\title{
IL PROGETTO BORBONICO DI UNA NAPOLI GIUDAICA TRA ANSIA DI RINNOVAMENTO E RITORNO AL PASSATO
}

\author{
THE BOURBON PROJECT OF A JEWISH NAPLES BETWEEN \\ THE ANXIETY OF RENEWAL AND A RETURN TO THE PAST
}

\author{
Maria Natale \\ Università Pegaso
}

\begin{abstract}
English: In Naples, since the early sixteenth century, a set of measures, characterized by variously afflictive contents, regulated the legal status of the Jews who had settled, also thanks to the protection offered to them by the Aragonese kings, in the southern part of the peninsula. The succession of these provisions testifies to the centrality of the Jewish theme in the complex balance of political, religious and economic relations but, at the same time, reveals the evident difficulty of finding a decisive answer to a question that, precisely by those forces, was constantly influenced. The outcome of this dynamism determined, over the centuries, the oscillation between admitting, although with different limitations, the settlement of the Jewish community and the need to decree its expulsion. Between the two poles, there was the imposition of various limitations including the obligation to wear the "sign".
\end{abstract}

Compared to these precedents, the edict about the readmission of the Jews, published in February 1740 by Charles of Bourbon, marked an epochal turning point. The objective pursued by the Bourbon government was not only to provide a base of support for commercial exchanges, but rather to create favourable conditions for the arrival and settlement of the Israelite community. At the basis of that edict there was the design of a Jewish Naples: a city capable not only of welcoming the Jewish community and allowing its peaceful establishment, but, above all, able to adopt the commercial and productive mentality of the Jews. That intellectual osmosis would have renewed the Kingdom.

Guaranteed by immunities and privileges, the Jews would have settled in Naples as had happened in Livorno, thanks to the so called Livornine. In Livorno, the Jews had become a very important component in the economic and political equilibrium. Thanks to the favourable initiatives taken by Ferdinando I de' Medici, the Israelite community had grown strong and prestigious and this had translated into an advantage for the overall economy. From a legal point of view, the Livornine became the archetype to be inspired by. This is confirmed by the frequent citation of the Livorno model in the eighteenthcentury sources concerning the kingdom of Naples. What happened in that city was the most effective term of comparison to demonstrate, against all prejudices, the usefulness of the Jews' peaceful settlement

A mercantilist vision pushed to consider the Jewish presence absolutely required. So it was necessary to overcome the widespread prejudices against a community of infidels and usurers. Adopted after a long and problematic debate, the Bourbon project required

* Italian Review of Legal History, 7 (2021), n. 5, pagg. 131-171

* https://riviste.unimi.it/index.php/irlh/index

* ISSN 2464-8914 - DOI 10.54103/2464-8914/16887. Articolo pubblicato sotto Licenza CC-BY. 
an act of courage. Thanks to it, the reformers carried out a regulatory intervention of sure innovation, but, above all, they showed, free from the interference of the Court of Rome, the ability and the strength to decide their own regulatory framework.

The reform did not represent a sectoral intervention, but it was part of a more complex legal plan aimed at cultivating human society. In this horizon, several sources record the link between that initiative and the contemporary institution of the Supreme Magistrate of Commerce. Launched in the space of a few months, both reforms were the result of the synergy between modern intellectuals and the governmental policy prevalent in the early years of the Bourbon kingdom. Any way the parallelism existing between the two historical events and their developments remarks an important change in the Charles' government. Both events followed a trend that, in time and manner, was comparable.

While developing within this common horizon, the Jewish affair was marked by undeniable specificities deriving from its political-religious implications. The tensions with the Church of Rome, already after the adoption of the edict, acted over time, tearing the basis of consensus for the reform. The effect was the gradual prevailing of a new prudent line of caution, that faithfully reflected the change taking place in the Court of Charles.

The progressive weakening of the Secretary of State José Joaquín de Montealegre and his definitive departure in 1746 radically changed the juridical-political horizon. In July 1747, the expulsion of the Jews from the Kingdom marked the decline of reformist actions.

Keywords: Jews; Charles of Bourbon; Supreme Magistrate of Commerce; infidels; ghetto

Abstract Italiano: La condizione giuridica degli ebrei, sin dai primi anni del XVI secolo, fu disciplinata a Napoli da un susseguirsi di provvedimenti dal contenuto variamente afflittivo. Rispetto a tali precedenti, la riforma varata da Carlo di Borbone segnò una svolta epocale. Adottato dopo una lunga e problematica gestazione, l'editto costituì parte di un più ampio quadro di riforme finalizzato a «coltivare l'umana società». In tale prospettiva, più fonti documentano il collegamento esistente tra quell'iniziativa e la coeva istituzione del Supremo Magistrato del Commercio. L'orizzonte comune ad entrambe le riforme risiedeva nella volontà di dare vita ad una nuova Napoli "giudaica", produttiva e commerciante: un'ambizione di rinnovamento che si infranse allorquando s'indebolì, e poi venne meno, la base di consenso e di sostegno politico che aveva trainato le riforme.

Parole chiave: Ebrei; Carlo di Borbone; Supremo Magistrato del commercio; infedeli; ghetto

Sommario: 1. Contro gli Ebrei «sin alguna exception». - 2. La ricerca di nuovi modelli. 3. Le iniziative "per promuovere l'umana società»: un orizzonte comune. -4 . Tenere il «piede fermo» e "pubblicare l'editto». - 5. Una difficile gestazione. - 6. A favore della «Nazione ebrea commerciante». - 7. Nuovi mercanti e antichi baroni. - 8. Riformatori a confronto: tra audacia e prudenza. -9. Parabole parallele. 


\section{Contro gli Ebrei «sin alguna exception»}

Sin dai primi anni del XVI secolo, a Napoli, la condizione giuridica degli ebrei fu disciplinata da una pluralità di provvedimenti. II ritmo serrato con cui tali disposizioni si avvicendarono offre conferma della centralità che il tema ebraico assunse rispetto al complesso intreccio dei rapporti politici, religiosi ed economici ma, al contempo, palesa l'evidente difficoltà di trovare una risposta risolutiva ad una questione che, proprio da quelle forze, era costantemente influenzata.

L'esito di tale dinamismo fu un'altalena normativa che, attraverso i secoli, oscillò tra l'ammettere, seppur con diverse limitazioni, lo "stabilimento» della comunità ebraica e l'esigenza di decretarne l'espulsione. Tra i due poli, ma il più delle volte come preludio della sanzione estrema dell'allontanamento, vi fu l'obbligo di "distinguersi», indossando un simbolo identificativo della propria appartenenza. II segno, diretto al precipuo scopo di evitare la periculosa commixtio tra ebrei e cristiani, rappresentò la sanzione più rilevante sotto il profilo simbolico, perché destinata a demarcare il confine, netto seppur ideale, tra la comunità dei cristiani e coloro che erano ad essa estranei.

In questa prospettiva, rilevante è il provvedimento con cui, il 12 gennaio 1509, tutti gli ebrei di età maggiore di dieci anni, «commoranti in qualsivoglia Città, Terra, Castello e luogo di questo Regno», erano obbligati a portare «il segno di panno rosso", sotto pena del pagamento di un'oncia d'oro per ciascuna infrazione. Un assunto lapidario interveniva a giustificare la norma: gli ebrei dovevano essere "appartati» ed "etiam divisi» dai cristiani, a cagione della loro «pravità Giudaica» ${ }^{1}$.

La disposizione colpiva una nutrita comunità ebraica che si era stabilita nella parte meridionale della penisola, anche grazie all'efficace protezione offerta dai sovrani aragonesi². Questi ultimi, infatti, attraverso plurime concessioni, avevano favorito il proficuo insediamento degli israeliti, attuando una politica che è stata spesso interpretata come il frutto di aperture precocemente illuminate, ma che $\mathrm{fu}$, più plausibilmente, l'esito di un ponderato calcolo economico e utilitaristico ${ }^{3}$.

L'assoggettamento di Napoli alla Spagna, mutando l'assetto complessivo dei poteri e dei rapporti, impose un decisivo cambio di direzione. II Mezzogiorno, antemurale dell'Impero spagnolo nel Mediterraneo contro gli infedeli, andava liberato, anche al suo interno, da tutti i nemici della cristianità. L'inflessibile visione iberica, contraria ad ogni forma di dissenso religioso, imponeva la lotta all'ebreo ${ }^{4}$. Qualsiasi forma di mediazione restava esclusa da quella logica stringente.

La rigidità dei precetti religiosi finì per plasmare l'azione politica, imponendo scelte inflessibili. Seguendo il ritmo di un vero e proprio crescendo normativo,

\footnotetext{
${ }^{1}$ Cfr. Prammatica I, De expulsione Hebraeorum sive ludeorum, in Giustiniani, 1804, p. 99.

${ }^{2}$ Del Treppo 1995, pp. 361-382

${ }^{3}$ Abulafia, 1998, pp. 35-53. Lacerenza, 2002, pp. 364-365.

${ }^{4}$ Lacerenza, 2017, pp. 141-142.
} 
l'obbligo di portare il segno anticipò, di poco più di un anno, il provvedimento di espulsione che fu emanato il 21 novembre del 1510. L'allontanamento, che sarebbe divenuto esecutivo nel marzo dell'anno successivo, veniva comminato sotto "pena de muerte y de confiscacion de todos sus bienes»: pene che sarebbero state inflitte «sin otro processo sentencia ni declaracion $»^{5}$.

Il bando recepiva di fatto le disposizioni con cui, diciotto anni prima, Ferdinando II d'Aragona ed Isabella di Castiglia avevano decretato l'espulsione degli ebrei dalla Spagna. Emanato il 31 marzo 1492, l'editto di Granada aveva inflitto un durissimo colpo alle comunità giudaiche presenti nei territori della Corona spagnola. Diretto ad attuare un radicale processo di epurazione della presenza israelita, il bando non contemplava alcuna possibilità di salvezza. Persino l'ipotesi della conversione al cristianesimo appariva soltanto teorica, giacché si comminava l'allontanamento anche ai novelli cristiani accusati di criptogiudaismo ${ }^{6}$.

Proprio per effetto di quelle disposizioni, molti ebrei, dopo essere stati scacciati dalla Spagna e poi dalla Sicilia ${ }^{7}$, avevano trovato rifugio a Napoli, città dalla quale dovettero presto andar via a causa del successivo bando di espulsione. La prammatica del 1510, se da un lato conteneva la rilevante rassicurazione de "levarse la Inquisitione da dicta Città et da tutto el regno predicto», dall'altro, quanto agli israeliti, infliggeva la sanzione estrema dell'allontanamento. Analogo provvedimento $\mathrm{fu}$, inoltre, emanato contro i neofiti, frequentemente accusati di doppiezza confessionale, i quali furono costretti ad abbandonare il Viceregno, conformandosi ad un ordine che venne anche ribadito nel $1515^{8}$.

Al di là delle sanzioni formali, che pure ebbero esecuzione, la persistente presenza degli ebrei nel Viceregno e la loro rilevante funzione economico-sociale costituiscono dati di fatto che giustificano l'avvicendarsi delle disposizioni.

Il 28 dicembre 1520, il Vicerè Raimondo de Cardona, recependo quanto «circa ludeos» era stato accordato da Carlo V a Madrid, consentiva agli ebrei di restare nel Viceregno, purché dietro pagamento di «mil y quinientos ducados de tributo ordinario». Veniva, inoltre, prevista la possibilità di un salvacondotto per "otras quarenta o cinquenta casadas ricas», capaci di versare il ridetto tributo ogni anno .

La disposizione, che manifestava la volontà di Carlo $\mathrm{V}$ di agire in una direzione diversa rispetto a quella assunta dal cattolicissimo nonno, alimentava l'altalena normativa di una spinta verso la tolleranza che si rivelava, però, debole ed incapace di resistere alle pressioni politico-religiose. Per i iodei napolitani, il 10 novembre 1539 giungeva, infatti, un nuovo ordine di espulsione. In attesa che

\footnotetext{
${ }^{5}$ Stock, 1977, pp. 32-35. Colafemmina, 2013, p. 36.

${ }^{6}$ Conde y Delgado de Molina R., 1991, pp. 41- 44.

${ }^{7}$ Pérez, 2005, pp. 221-266. Fiume, 2009, pp. 4-5.

${ }^{8}$ Stock, 1977, p. 33. Colafemmina, 2013, pp. 26-28.

9 II documento è pubblicato in appendice a Colafemmina, 2013, p. 39-42. La citazione è a p. 40 .
} 
il provvedimento avesse avuto concreta esecuzione, si disponeva che dovesse essere assegnato alle famiglie israelite un "locus vel platea separata», affinché vi abitassero senza «indecentia facinora producere». Allo scopo di evitare la ben nota periculosa commixtio, era loro ordinato di indossare un «signum coloratum, et notabile»: i maschi avrebbero calzato un berretto di colore rosso, o anche croceo o giallo, mentre le donne avrebbero portato una "fasciam ejusdem coloris»" .

Anche in tal caso, le disposizioni che imposero il signum spalancarono le porte al successivo provvedimento di espulsione. Infatti, nel Maggio del 1541 la cacciata definitiva fu opera di Don Pedro de Toledo che impose agli ebrei di abbandonare il Viceregno nel termine di quattro mesi, sotto pena di confisca dei loro beni e con facoltà di potervi rientrare soltanto in occasione delle fiere ${ }^{11}$.

È da notare che, al di là del piano delle affermazioni esplicite, l'iniziativa di espulsione adottata da Don Pedro de Toledo si inseriva appieno nella dialettica socio-istituzionale napoletana.

A distanza di circa due secoli da quell'evento, la riflessione di un acuto osservatore, qual era Pietro Contegna ${ }^{12}$, ci restituisce i contorni di un provvedimento che si inseriva appieno in quella "rivoluzione istituzionale, politica, cetuale» che avvenne, in modo graduale, nello Stato napoletano tra il 1524 ed il 1542 e che portò all'espulsione della nobiltà dal governo ${ }^{13}$. Nell'intento di ricondurre, sotto l'unico ordine monarchico, un corpo sociale sottomesso ad una pluralità di poteri particolaristici, si decideva di intervenire, in più modi, per ridurre il potere di una nobiltà riottosa ed ostile ad adeguarsi ai cambiamenti già in atto nelle grandi monarchie territoriali d'Europa.

In questo quadro, Contegna coglieva il significato più profondo e, certamente meno evidente, del provvedimento di espulsione degli ebrei:

è noto poi che gli ebrei furono cacciati da Napoli sotto l'Imperador Carlo Quinto forse, e senza forse, per lo dispetto che volle fare alla Nobiltà Don Pietro Toleto allora Vicerè di Napoli; giacché per le caggioni note a tutti coloro che anno cognizione della Storia Napoletana, essendo divenuto implacabile nemico della nobiltà suddetta, procurò il discacciamento degli ebrei dal Regno più tosto per fare danno alla Nobiltà debbitrice degli ebrei di molte somme e che dovendo costoro partire dovevano immediatamente essere soddisfatti, che per evitare le usure le quali da questi che si commettevano, giacché era facile il dar rimedio a questo male per altra via; come infatti vi danno rimedio tutti gli altri Principi Cattolici e gli stessi Sommi Pontefici che tengono gli ebrei nelli Stati loro ${ }^{14}$.

10 Prammatica II, De expulsione Hebraeorum sive ludeorum, in Giustiniani, 1804, p. 99.

${ }^{11}$ Abulafia, 1997, pp. 5-44.

12 Su Pietro Contegna, Ajello, 1980, pp. 383-412; Cernigliaro, 2006, pp. 170-181; più di recente, Trifone, 2013, ad vocem ed i recenti approfonditi studi di Luongo, 2018, pp. 373482.

${ }^{13}$ Su questi temi cfr. Cernigliaro, 1984; Cernigliaro, 1998; Ajello, 1998; Del Bagno, 2000, pp. 172-173.

${ }^{14}$ Archivio di Stato di Napoli (d'ora innanzi ASN), Ministero degli affari esteri, 4401, 6/9, 
L'espulsione, andando ad incidere direttamente sui meccanismi economici e finanziari che reggevano l'economia regnicola, era stata utilizzata quale strumento di attacco al potere baronale. Il prestito ad usura, cui anche la nobiltà faceva ampio ricorso, si era rivelato il tallone d'Achille da colpire per indurre quella riottosa componente cetuale a piegarsi.

In questo quadro, i richiami, frequenti nelle fonti cinquecentesche, agli inconvenienti, ai pericoli ed agli scandali derivanti dal contatto con i giudei lasciano intendere la volontà di far presa su moventi, per molti versi anche istintivi e passionali, in grado di celare sottotraccia le reali finalità politico-istituzionali e di consentirne, per questa via, il perseguimento. Al di là delle affermazioni di principio, che il più delle volte appaiono rivolte a stigmatizzare sul piano giuridico l'inflessibilità della lotta contro il dissenso religioso, è il difficile equilibrio tra le forze in gioco a dare consistenza alla norma. Così, se in linea di principio, i provvedimenti contro gli israeliti non ammettono mezzi termini, dall'altro il loro succedersi conferma che, al di là delle sanzioni formali, il problema ebraico continuava a essere questione su cui era opportuno ritornare, quasi a dover continuamente ricalibrare lo sforzo giuridico.

II 17 luglio 1572, un trentennio dopo il provvedimento di espulsione toledano, al fine di evitare gli «scandali» e gli inconvenienti derivanti dal contatto con $\mathrm{i}$ mercanti ebrei presenti nel Regno in occasione delle fiere, era prescritto a questi ultimi l'obbligo di portare il berretto di panno giallo, al fine di essere «conosciuti da tutti per Ebrei come sono, sotto pena di cinque anni di galea e di altra pena corporale $\aleph^{15}$. Anche in tal caso, la norma poneva, quale premessa della sanzione, considerazioni di tipo socio-religioso che trovavano una base di consenso nell'insofferenza diffusa contro la comunità giudaica. II governo dimostrava di voler attingere a quegli umori legittimandoli nella realtà giuridica.

Nel corso del Seicento, le voci ricorrenti a proposito degli ebrei quali untori della peste recano conferma della persistenza di un clima di odio e di generale ostilità che continuava a fare da costante sfondo alle disposizioni afflittive.

Per questa via, due secoli dopo il provvedimento di espulsione emanato da Don Pedro de Toledo, una nuova prammatica interveniva senza mezzi termini anche contro i mercanti giudei, che fossero impegnati nel Regno in occasione delle ferie o con licenza di negoziare, per imporre loro di «uscire dal presente Regno dentro il termine di giorni quindeci», "sotto la pena della confiscazione di tutti li loro beni [...] $]$ ed anche di carcerazione» ${ }^{16}$.

La prammatica, emanata il 29 aprile 1702, riproponeva, con ancor più asprezza, le ragioni dell'espulsione. II 13 marzo 1708, l'ordine di «sfrattare» dal Regno era di fatto rinnovato, comandando che «en el termine de ocho dias», fossero sfrat-

\footnotetext{
Riflessioni su la lettera venuta di Roma in proposito degli ebrei.

${ }^{15}$ Cfr. Prammatica III, De expulsione Hebraeorum sive ludeorum, in Giustiniani, 1804, pp. 100-101.

${ }^{16}$ Cfr. Prammatica IV, ivi, p. 101.
} 
tati «de este Reyno todos los Ebreos que estan en esta Capital, y commoranen el Reyno sin alguna exception ${ }^{17}$.

\section{La ricerca di nuovi modelli}

L'orientamento contra iudeos, che a Napoli resistette, pur tra alterne vicende, sino alla svolta impressa dal governo carolino nel 1740, dà conto soltanto di uno dei diversi approcci che, nella penisola, si delinearono riguardo al tema della presenza ebraica ${ }^{18}$. Gli atteggiamenti dei governi furono, in tal senso, ampiamente diversificati in ragione delle differenti condizioni politico-religiose ed, in misura ancor più rilevante, della diversa considerazione attribuita alla Nazione ebrea quale attore economico.

In un panorama giuridico, qual era quello di Antico Regime, caratterizzato dalla frammentarietà e disomogeneità della soluzioni giuridiche, la disciplina relativa alla condizione degli ebrei si connota per una vera e propria "scomposizione dei percorsi storici in mille rivoli» ${ }^{19}$, caratterizzata, salvo alcune eccezioni, dalla precipua volontà di conservare una rassicurante uniformità a livello sociale e, prima ancora, sul piano religioso. La disciplina della materia, infatti, imponeva di tener conto di una ricca ed ampia serie di questioni soggette al magistero della Chiesa, che esercitava, pertanto, un'influenza fortissima sugli orientamenti della politica e sulle scelte concrete adottate dai sovrani. L'orizzonte vario ed articolato delle soluzioni giuridiche rispecchiava un innegabile complesso dinamismo fortemente connotato dalle specificità delle situazioni locali e dalla peculiare condizione che l'ebreo assumeva, anche rispetto agli altri stranieri² ${ }^{20}$

I sentieri, spesso ambigui e tortuosi, attraverso i quali si concretizzò l'impegno di ostacolare qualsiasi forma di dissenso religioso, non impedirono lo sviluppo di ambiti in cui riuscirono a prevalere orientamenti di apertura. Tuttavia, anche in tali contesti, piuttosto che elaborare soluzioni generali, i sovrani scelsero di percorrere strade diverse, attuando la tolleranza di fatto o utilizzando la categoria giuridica del privilegio ${ }^{21}$. La scelta di queste soluzioni rappresenta di per sé un elemento significativo: entrambe, seppure con una diversa gradualità, manifestano l'oggettiva difficoltà di ammettere, in linea di principio, la possibilità di una convivenza, pacifica e giuridicamente garantita, con l'infedele.

Ad indurre verso soluzioni di favor nei confronti della comunità ebraica erano motivazioni di ordine economico: la possibilità di disporre dei capitali che erano nella mani dei prestatori israeliti costituiva un obiettivo allettante per molti governi. A ciò si collegava il proposito di conferire vivacità ai traffici commerciali e di

\footnotetext{
${ }^{17}$ Cfr. Prammatica V, ivi, pp. 101-102.

${ }^{18}$ Caffiero, 2014.

${ }^{19}$ Edigati, 2020, p. 927.

${ }^{20}$ Storti Storchi, 2012, pp. 127-128.

${ }^{21}$ Edigati, 2020, p. 930.
} 
promuovere gli scambi. In questa prospettiva, diventava essenziale puntare sulla componente ebraica, quale fulcro vitale capace di catalizzare l'attività finanziaria ed economica.

Gli esiti positivi di questi orientamenti erano evidenti in diversi contesti territoriali della penisola, soprattutto nelle città portuali che erano, per loro natura, più aperte. In alcune, come Trieste, Venezia, Pisa e Livorno, l'adozione di provvedimenti favorevoli alle comunità ebraiche era stato motivato dall'attenzione costante alle dinamiche del commercio, dalla necessità di garantire la circolazione di ingenti capitali e dalla volontà di far parte di reti commerciali transnazionali ${ }^{22}$. In questo quadro, il modello più significativo era offerto dalla città di Livorno, dove gli Ebrei erano diventati una componente numerosa e di sicuro rilievo negli equilibri economici e politici. Grazie alle iniziative assunte da Ferdinando I de' Medici, la comunità israelita era cresciuta forte e prestigiosa e ciò si era tradotto in un vantaggio per l'intera città e per l'economia complessiva.

II valore paradigmatico delle Livornine, emanate dal granduca Ferdinando I de' Medici il 30 luglio 1591 e poi, con alcune aggiunte e modifiche, il 10 giugno 1593, stava nella capacità di concepire un regime giuridico fondato sulla tolleranza, pur nell'ambito di un ordinamento cattolico ${ }^{23}$. L'obiettivo perseguito dal governo mediceo non era stato soltanto quello di fornire una base di appoggio per gli scambi commerciali, piuttosto quello di favorire l'arrivo e l'insediamento di una comunità che era garantita, sul piano personale e patrimoniale, da una serie di vantaggi che per certi aspetti la collocavano in una posizione più favorevole rispetto agli autoctoni ${ }^{24}$.

Gli effetti di quelle disposizioni non avevano tardato a rendersi evidenti: studi demografici calcolano che nella sola Livorno, la comunità ebraica crebbe rapidamente. Agli inizi del Seicento era limitata ad un centinaio di unità, contava a metà Settecento circa cinquemila persone destinate ad incrementarsi a settemila sul finire del secolo ${ }^{25}$.

Era diventata convinzione diffusa che la prosperità di Livorno fosse direttamente riconducibile alle disposizioni grazie alle quali la comunità israelita aveva nel tempo prosperato. II regime privilegiato concesso agli Ebrei era, infatti, coinciso con la creazione medicea di una città all'avanguardia per i tempi e destinata ad essere il grande porto della Toscana, scalo preferenziale per molte marinerie del Nord $^{26}$.

\footnotetext{
22 In questa prospettiva, per Trieste, si cfr. Dubin, 1999; Gatti, 2008 e Dubin, 2010; per Pisa e Livorno, L. Frattarelli Fischer, 2008 e, più di recente, gli studi di Edigati, 2020 e Edigati, 2021; per Venezia, cfr., in particolare, Ravid, 1978 e Ravid, 2018 (1a 2003).

${ }^{23}$ Sul tema si cfr. la ricca ed accurata analisi di Edigati, 2021, pp. 45-77. Sulle Livornine si cfr. l'edizione critica curata da Frattarelli Fischer, 2019.

${ }^{24}$ Edigati, 2020, p. 949.

${ }^{25}$ Giura, 1977, p. 5; Galasso, 2002, pp. 1-174.

${ }^{26}$ Sul punto cfr. Cassandro, 1984, pp. 567-569.
} 
Fu questo complesso di fattori che consacrò Livorno a modello. Dal punto di vista giuridico, dunque, le Livornine divennero l'archetipo cui ispirarsi.

Ne reca conferma la frequente citazione del modello labronico nelle fonti settecentesche riguardanti il regno di Napoli. Quanto accaduto in quella città costituiva il più efficace termine di paragone per dimostrare, contro ogni pregiudizio, l'utilità del «pacifico stabilimento» degli ebrei.

Il fatto stesso che i provvedimenti medicei fossero diventati a Napoli l'archetipo normativo dei riformatori stigmatizza il mutamento del loro orizzonte giuridico. Una visione utilitarista e mercantilista spingeva a considerare necessaria la presenza giudaica e di conseguenza, non senza difficoltà, induceva a relegare al margine ogni altra preoccupazione. Le ragioni dell'economia imponevano di superare secolari barriere ed agivano nel tentativo di declassare a pregiudizio l'inveterata ostilità religiosa.

Si trattava di un modo inconsueto di approcciare al problema che, a Napoli, riuscì a manifestarsi, in forme più o meno esplicite, soltanto all'indomani dell'indipendenza del Regno, allorquando una straordinaria congiuntura storica determinò le condizioni favorevoli al prevalere degli orientamenti culturali dei giuristi novatores. La loro sensibilità all'ideologia economica trovò terreno fertile nella nuova situazione politica, che, mutando il rapporto delle forze internazionali, era destinata ad avere ripercussioni rilevanti anche nei confronti dalle grandi potenze marittime.

La svolta dinastica alimentò le speranze di cambiamenti strutturali, capaci di intaccare il nocciolo duro, e per molti versi incancrenito, di una società bloccata qual era quella napoletana. La conquistata indipendenza nutrì nei riformatori la fiducia in progetti di rinnovamento che, grazie al "proprio Principe» ${ }^{27}$, avrebbero riscattato un paese prostrato da secoli di dominazioni straniere.

Il complesso delle nuove condizioni politico-istituzionali rassicurava gli intellettuali più critici e moderni e li spingeva a credere che fosse giunto il momento di sacrificare gli interessi dei ceti tradizionalmente dominanti e di tentare un'operazione di rinnovamento a dir poco complessa.

In questa prospettiva, è un dato significativo che le problematiche relative a «li infedeli», ossia a coloro che «non pratticavano la SS.ma Religione, il culto e l'ossequio verso il nostro Dio», diventassero il fulcro di una delle prime Consulte della Giunta di commercio. L'organismo, istituito quale sede privilegiata di discussione su temi economici e finanziari, era stato introdotto con dispaccio del 16 aprile 1735, ma era diventato operativo solo nel febbraio del $1736^{28}$. II 30 aprile dello stesso anno, la Giunta si apprestava a discutere un tema di rilevanza fortissima con dirette ripercussioni sui rapporti con la Chiesa.

Al centro del dibattito vi era, infatti, se convenisse «procurar la Pace co' Turchi

\footnotetext{
${ }^{27}$ La citazione è tratta dalle Considerazioni intorno al commercio del Regno di Napoli di Gregorio Grimaldi, pubblicate in appendice a Pilati, 2001, p. 319.

${ }^{28}$ Sulla Giunta di commercio borbonica, rinvio a Natale, 2014, pp. 137-159.
} 
d'Africa o co' Turchi solo, o pure né con gli uni, né con gli altri», ma il parere è significativo di una presa di posizione più generale. Ponendo in diretta correlazione «li pregiudizi della Ss.ma Religione» e il "comodo de' Popoli», i membri della Giunta si esprimevano tracciando uno spartiacque tra «il culto e l'ossequio, verso il nostro Dio», ed il «detrimento» causato da scelte economiche e politiche basate sulla cieca obbedienza religiosa ${ }^{29}$. La disciplina dei rapporti con gli infedeli non poteva subire i condizionamenti derivanti da una visione miope ed assorbente della religione:

Imperocché la nostra SS.ma Religione è tutta uniforme al dritto della Natura, da cui siamo stimolati a coltivar l'umana società, e che il Commercio con gente, benché di diversa e perciò falsa Religione, seco non porta l'approvare l'altrui errori e follie, ma una comunicazione di quei scambievoli uffizj, che son proprj degli uomini, come uomini ${ }^{30}$.

Qualsiasi disposizione avesse ammesso o, ancor meglio, promosso rapporti commerciali con gli infedeli non avrebbe violato alcuna legge, perché le «Sacre Pagine non contenean questa proibizione di aver pace e commercio con gente di falsa religione $»^{31}$.

Ispirandosi al modello offerto da altri Stati, bisognava regolamentare quelle relazioni rimuovendo ogni riserva o condizionamento, al solo scopo di promuovere le migliori condizioni di produttività.

Il realismo politico imponeva l'adozione di misure chiare e stringenti. Tuttavia, sul piano esplicito delle dichiarazioni, la radicale originalità delle soluzioni spesso era costretta ad indulgere verso giustificazioni di natura retorica, che erano indirizzate a trovare il consenso dei molti scettici e conservatori. Da questa esigenza traevano origine i frequenti richiami alla necessità di realizzare una convivenza con gli infedeli, al solo fine di poter sperare nella loro conversione alla religione cristiana, grazie alla «caritatevole persuasione» e all' «esempio degli uomini pii e divoti» ${ }^{32}$.

Abbattere le frontiere dettate dai pregiudizi avrebbe provocato la possibilità di evangelizzare attraverso l'esempio: una motivazione diretta a legittimare scelte che muovevano, in realtà, da una nuova visione dell'economia e della società e che avrebbero comportato, seppure indirettamente, un inserimento, quanto mai difficile e limitato, degli ebrei nelle società ${ }^{33}$.

\footnotetext{
${ }^{29}$ Società napoletana di Storia patria (d'ora innanzi citata come SNSP), ms. XXI d30, cc. 9r. e ss.

${ }^{30}$ Ivi, c. $16 r$.

${ }^{31}$ Ivi, cc. 16r. e v.

32 Ivi, c. $17 v$.

${ }^{33}$ A. Foa, 2001, p. 5; Tasmani, 1999, pp. 187-220; Eisentadt, 1993.
} 


\section{Le iniziative "per promuovere l'umana società»: un orizzonte comune}

Le iniziative riformistiche assunte nei primi anni di dominazione borbonica trassero linfa vitale dalla fiducia di poter stimolare, attraverso un generale processo di rinnovamento, le condizioni per «promuovere l'umana società » ${ }^{34}$. Tale espressione era efficacemente utilizzata proprio dai membri della Giunta di commercio carolina per sostenere due innovazioni dal forte impatto socio-economico: l'apertura del commercio con il Levante e l'insediamento degli ebrei entro i confini del Regno.

Appare significativo che l'ipotesi di una riammissione dei giudei venga formulata congiuntamente a quella relativa all'avvio dei traffici con l'Oriente: ambedue le questioni imponevano di considerare, in modo nuovo, il rapporto con gli infedeli, alla luce di preminenti istanze economiche. In entrambi i casi, l'elaborazione della strategia giuridico-politica imponeva di tener conto delle numerose variabili in competizione, attribuendo valore prioritario a ciò che giovava alla produttività.

In questa prospettiva, da un canto, la posizione privilegiata del Regno nel Mediterraneo avrebbe garantito un commercio florido con l'Oriente, dall'altro, l'introduzione degli ebrei, da sempre versati negli affari e forniti di ingenti capitali, avrebbe immesso in circolazione la liquidità necessaria per l'adeguato sviluppo dell'economia regnicola.

Lungi dal rappresentare degli interventi di settore, dunque, le disposizioni riguardanti la comunità israelita sono in grado di restituirci la cifra di quel mutamento di prospettiva che s'ispirava ad una generale tendenza innovatrice e che mirava ad investire ogni campo.

In tal senso, appare significativa la congiuntura temporale che lega, altresì, le iniziative riguardanti gli infedeli e l'introduzione del Supremo Magistrato del Commercio ${ }^{35}$.

Il collegamento esistente, sin dal nascere, tra le iniziative è testualmente dimostrato dalla corrispondenza, avvenuta tra l's ed il 12 ottobre 1739, tra José Joaquín de Montealegre e Pietro Contegna. II Segretario di Stato incaricava l'abate di Arienzo di «mettere in ordine ed in stato di pubblicarsi li reali sapientissimi secreti sull'erezione delli Magistrati del Commercio» e, al contempo, di esprimersi, congiuntamente a Celestino Galiani ${ }^{36}$, in ordine all'introduzione degli Ebrei nel regno di Napoli e di Sicilia ${ }^{37}$.

Nell'autunno del 1739 l'editto istitutivo del Supremo Magistrato del commercio

\footnotetext{
${ }^{34}$ SNSP, ms. XXI d30, Parere della Giunta di commercio del 30 aprile 1736.

${ }^{35}$ Sulla magistratura commerciale Tita, 2012; Ciancio, 2012; Natale, 2014.

${ }^{36}$ Un sintetico, ma significativo, giudizio su Celestino Galiani è espresso in una lettera autografa del 29 luglio 1724 scritta a Bottari da Antonio Niccolini, che lo definisce «veramente uomo incomparabile». Biblioteca Corsiniana di Roma, cod. cors. 1891 ms. 44.E.1, c. 20. Su Galiani, cfr. Origlia, 1753, pp. 248-257; Nicolini, 1951; Ferrone, 1982; GalianiGrandi, 1989; Natale, 2002, pp. 115-162.

${ }^{37}$ ASN, Ministero degli affari esteri, 4400, 9/4.
} 
condusse alla creazione di un tribunale dai caratteri radicalmente innovativi con la finalità di adeguare la giustizia, in ambito commerciale, a nuovi canoni di produttività giudiziaria. Celerità, economicità e semplificazione furono gli obiettivi di questa riforma giudiziaria che, attraverso l'adeguata tutela dei rapporti giuridici anche nella loro fase patologica, mirava a favorire le relazioni commerciali.

L'introduzione del Supremo Magistrato precedette di pochi mesi il provvedimento di ammissione degli ebrei che fu varato, infatti, nel febbraio dell'anno successivo.

In perfetta connessione temporale ed ispirato alle medesime finalità, fu il progetto di aprire il commercio con il Levante che si concretizzò il 7 aprile 1740 con la conclusione del trattato con la Porta sottoscritto da Giuseppe Finocchietti Faulon. Nonostante le grandi speranze dei riformatori, in realtà, anche quel trattato si rivelò presto inefficace, vista la ferma opposizione della Francia, che non intendeva cedere la posizione di assoluto predominio esercitata su quei mari ${ }^{38}$.

Al di là degli esiti, tutte le iniziative, che appaiono collegate tra loro da un evidente fil rouge, marcano il progressivo delinearsi di un quadro che, frutto dei nuovi orientamenti intellettuali progressisti manifestatisi nei primi anni del governo carolino, mirava ad incidere sulle condizioni materiali del Regno.

\section{Tenere il "piede fermo» e "pubblicare l'editto»}

L'orizzonte comune in cui si sviluppano le diverse, ma coerenti, iniziative di riforma non impedisce di rimarcare le specifiche implicazioni che la proposta di riammissione degli ebrei portava con sé. Ad essere in discussione non era soltanto la possibilità di realizzare un intervento normativo di sicura innovazione rispetto al passato, ma, soprattutto, la forza di affermare l'autonomia decisionale del Regno, dando vita ad un assetto normativo indipendente dalle ingerenze della Chiesa. Per agire compiutamente in tale direzione, era necessario intervenire sulla materia ebraica senza mezzi termini: ad avviso di Contegna, infatti, la Corte di Roma era "simile ad un fantasma; perseguita chi la teme, e fugge, e sparisce, innanzi a colui che tiene il piede fermo ${ }^{39}$. Bisognava procedere e «pubblicare l'editto nelle forme che bisogna ${ }^{40}$ in un momento che, evidentemente, visto da Napoli, doveva apparire favorevole ad ultimare l'impresa. A Roma, infatti, in un clima dominato da una sostanziale stasi e dall'incertezza per il futuro, si stavano consumando gli ultimi giorni di papa Clemente XII, la cui morte, attesa da tempo, giunse il 6 febbraio 1740, tre giorni dopo la pubblicazione a Napoli del bando sulla riammissione degli ebrei.

\footnotetext{
${ }^{38}$ Ajello, 1976, p. 656.

${ }^{39}$ ASN, Ministero degli affari esteri, 4401, 6/4, Lettera del Contegna del 30 gennaio 1740 a riguardo della memoria del Cardinal Firrao. Per la memoria del Cardinale Firrao inviata da Roma il 22 gennaio 1740, si cfr. ASN, Ministero degli affari esteri, 4401, 6/3. ${ }^{40}$ Ibidem.
} 
La straordinaria congiuntura temporale in cui si colloca la proclamazione del salvacondotto carolino accredita la tesi della centralità del problema ebraico nella dinamica complessiva dei rapporti tra Napoli e Roma. È noto che all'indomani della conquista, i rapporti con la Santa Sede avevano costituito per il giovane monarca il primo e il più rilevante dei problemi ${ }^{41}$. La diplomazia romana aveva cercato, dopo ventisette anni di governo austriaco, di trarre vantaggio dalla svolta dinastica: lo scopo era stato sempre, più o meno dichiaratamente, quello di affermare una politica pontifica orientata alla difesa delle prerogative ecclesiastiche ${ }^{42}$.

Le alterne vicende che avevano contrassegnato la faticosa ricerca di un «accomodamento" nei rapporti tra Napoli e Roma erano state, via via sempre più marcatamente, contrassegnate dall'operato dei medesimi protagonisti della contesa ebraica: Celestino Galiani e Pietro Contegna. Recenti ricerche documentano che proprio il sacerdote di Arienzo fu «tra gli intellettuali meridionali del primo Settecento maggiormente sensibili alle istanze riformatrici»: strenuo difensore delle tesi regie, si adoperò, da ecclesiastico, per ridurre i privilegi del proprio ceto, indirizzando l'azione politica verso il contenimento delle immunità ecclesiastiche, come aspetto del più ampio progetto di compressione delle manifestazioni del particolarismo cetuale ${ }^{43}$.

II vero arbitro del Concordato, che fu raggiunto nel 1741, fu però Celestino Galiani $^{44}$. Grazie alle propria fama ed alla ben nota capacità di mediazione, il Cappellano Maggiore era entrato a far parte della giunta giurisdizionale nominata all'indomani dell'arrivo di Carlo a Napoli e, col tempo, era divenuto il principale attore della complessa vicenda, essendo stato inviato a Roma, già nel luglio del 1738 , allo scopo di riprendere le trattative ${ }^{45}$.

Nell'estate di quello stesso anno, anche un altro evento aveva giocato un ruolo fondamentale per imprimere una svolta ai negoziati con Roma: la decisione della Corte di Spagna di destituire il segretario di Stato Manuel Domingo de Benavides y Aragón, conte di Santisteban, e di porre al vertice del governo napoletano José Joaquín de Montealegre. Il cambio di guardia aveva determinato un indirizzo radicalmente diverso anche nei rapporti con la Santa Sede: sin da subito, infatti, il nuovo Segretario di Stato aveva assunto un atteggiamento più risoluto nei confronti della Chiesa di Roma.

Delineatosi il nuovo orientamento, erano mutate comprensibilmente le strategie. Così, mentre la parte borbonica, forte del sostegno del Segretario di Stato, aveva tentato di accelerare il processo negoziale affidandosi, nel concreto, alle doti mediatrici del Cappellano Maggiore, la curia romana aveva ripiegato su un atteggiamento indolente che era il chiaro indice di una tattica dilatoria che mirava

\footnotetext{
${ }^{41}$ Ajello, 1976, p. 489 e ss.

${ }^{42}$ Cfr. Caridi, 2011, p. 535.

${ }^{43}$ Luongo, p. 373 -386. La citazione è a p. 373.

${ }^{44}$ Novi Chavarria, 2020, pp. 11 e 120.

${ }^{45}$ Ajello, 1976, p. 649.
} 
a vedere realizzato dapprima il ricambio sul soglio pontificio. É comprensibile che la Corte di Roma non volesse assumere, in un momento di oggettiva precarietà, il peso di un accordo con Napoli che avrebbe comportato un ridimensionamento delle prerogative godute fino ad allora dalla Chiesa in quel Regno ${ }^{46}$.

Se, dunque, la politica dilatoria posta in essere dalla Chiesa confermava l'evidente preoccupazione di Roma di dover cedere delle posizioni, era necessario approfittare della fragilità del momento per assumere, a Napoli, le decisioni che più erano condizionate dalle pressioni ecclesiastiche: la questione ebraica era certamente una di queste.

L'invito di Contegna a tenere «il piede fermo» pubblicando l'editto di riammissione va letto in questa prospettiva: il momento appariva particolarmente propizio per varare un provvedimento che, era già di per sé, ampiamente divisivo.

In tal senso non poteva tacersi che, mentre vi erano coloro che non nutrivano alcun dubbio circa l'utilità dello "stabilimento degli ebrei», ritenendolo "fra gli strumenti stimati di ristabilire e ravvivare il commercio di questi Regni delle due Sicilie», altri evidenziavano la pericolosità insita in una comunità che era per lo più dedita al prestito ad usura ${ }^{47}$.

In questa prospettiva, l'ingresso di gente "potenziosa in denaro contante» spaventava quanti ritenevano che gli Ebrei, "già introdotti per l'addietro in questa città», vi erano stati cacciati proprio "a cagione dell'usura esorbitante» e che non vi erano stati «riammessi, attesi i mali che avevano precedentemente infetti» ${ }^{48}$.

L'usura costituiva, dunque, un punto nodale della contesa. L'immagine degli ebrei-prestatori di denaro, generava una reazione d'immediata repulsione e di diffusa preoccupazione

Il circolo vizioso instaurato dal divieto di possedere beni immobili e d'impegnarsi in altre utili attività economiche aveva, di fatto, obbligato gli ebrei alla pratica dell'usura, ma ciò aveva accentuato i toni della già irremovibile condanna cristiana ${ }^{49}$.

Da sempre, la disputa sulla liceità del prestito ad interesse era stata alimentata dalle prescrizioni bibliche, che avevano diffuso l'idea che, in linea di principio, non fosse in discussione la misura del corrispettivo, ma persino la liceità di prevederne e di contrattarne uno qualsiasi. Tuttavia, se la norma fosse stata applicata

\footnotetext{
${ }^{46}$ Roma riuscì nell'intento di ritardare l'approvazione del concordato con Napoli. La sua maturazione si rese possibile solo all'indomani della morte di Clemente XII e grazie all'ascesa al soglio pontificio di Benedetto XIV, al secolo Prospero Lambertini. II nuovo Pontefice salì al soglio pontificio nell'agosto del 1740 ed il trattato di accomodamento tra la Santa Sede e la Corte di Napoli fu concluso in Roma e ratificato a Napoli l'8 giugno 1741 e a Roma il 13 giugno.

${ }^{47}$ II 17 giugno 1739 i motivi della contesa erano racchiusi in un documento Circa gli ebrei in cui erano riassunti i caposaldi della questione. Si cfr. ASN, Ministero degli esteri, 4400, $1 / 3$.

48 Ibidem.

${ }^{49}$ Todeschini, 2003, p. 106 e ss.
} 
com'era scritto nelle Sacre scritture ${ }^{50}$, ne sarebbe seguita la paralisi del traffico economico non solo dei privati, ma anche degli Stati, la cui economia si fondava in gran parte sul debito pubblico, ossia sui prestiti ad interesse dei sudditi a favore del fisco.

L'esistenza stessa di questa contraddizione aveva generato accorgimenti mediativi di carattere pragmatico, spesso tortuosi: soluzioni capaci di realizzare in fatto ciò che in teoria era condannato, conciliando il rispetto delle forme ideali con la soddisfazione delle esigenze materiali. Nel tempo si erano imposti complicati aggiustamenti tra due opposte spinte: le esigenze dell'economia, di regola già prostrata, e la necessità di reprimere l'usura, reato che proprio dal disastro della produttività trovava alimento.

L'usura rappresenta il caso emblematico in cui la logica medievale, non potendo in concreto render esclusiva ed operativa la preclusione testuale evangelica contraria all'esazione di qualsiasi interesse sui prestiti di danaro, era costretta ad esercizi funambolici per poter conciliare la lettera del testo con le esigenze materiali ${ }^{51}$. II ricorso a schemi contrattuali, anche solo formalmente diversi dal mutuo, richiamato testualmente nel Vangelo di Luca e per sua natura gratuito, era stata soluzione capace di realizzare l'equilibrata mediazione tra l'osservanza delle proibizioni canoniche e la soddisfazione delle esigenze del mondo mercantile.

Così la normativa in materia di usura si era andata stratificando come frutto di quel complesso intreccio tra etica e diritto, tra ragione e religione: poli tra loro difficilmente conciliabili se non a patto di accomodamenti formali, capaci di quietare, nel contempo, i rimorsi dell'anima ed i bisogni del corpo. La prassi giuridica aveva nel tempo elaborato una casistica contrattuale capace di soddisfare le esigenze dell'economia assicurando la circolazione monetaria e garantendo lo sviluppo degli affari, fermo restando il rispetto formali dei precetti divini che da quelle conciliazioni pratiche non erano affatto scalfiti. Sul versante pratico, infatti, la stessa Chiesa aveva predisposto strumenti deputati a non bloccare il dinamismo mercantile. L'ammissione degli Ebrei nei territori della Chiesa Romana aveva risposto alla medesima logica e questo dato testimoniava l'ambiguità delle posizioni ecclesiastiche, inclini ad ammettere sul piano pratico quanto poteva restare proibito sul piano ideale.

In questo quadro, in cui teoria e prassi seguivano sentieri diversi, gli ebrei, prestatori di denaro ed usurai, restavano i destinatari della più ampia riprovazione formale. Da sempre, contro di essi, si levava il più severo biasimo che trovava sfogo in un diffuso sentimento di condanna.

Un geniale illuminista come Antonio Genovesi, nelle sue "Considerazioni su i fondamenti della civile società o sulle leggi dei corpi politici", osservava:

\footnotetext{
${ }^{50}$ Sui rapporti tra usura e cristianesimo cfr. Nelson, 1967; Boari M., 1992, XLVI, pp. 11381139; Le Goff, 1986, pp. 15-17; i contributi raccolti da Macario F. Manna A. (ed), 2002; Gamba, 2003; Tita, 2008.

${ }^{51}$ Santarelli, 1992, pp. 153-155.
} 
Confessiamo nondimeno che, benché i Giudei fossero rei di non legittime usure, quasi tutte le leggi emanate contra di essi sentono piú d'invidia e d'odio pubblico, che abbian di setata ragione. La legge non debbe incollerirsi; ella è ragione, non passione ${ }^{52}$.

Nel tempo, irrazionalità e odio avevano inquinato il diritto. Ne era derivata una normativa che, incollerita dall'invidia e dall'eccesso di passione, era divenuta interprete dei sentimenti più volgari.

La capacità finanziaria della comunità ebraica e la centralità assunta nella circolazione del denaro avevano funzionato da spinta catalizzatrice di un'onda montante di odio che si era riversata, più o meno consapevolmente, nella disciplina della condizione giuridica degli ebrei. Ne derivava un'ulteriore considerazione, che da Genovesi era stata soltanto evidentemente accennata: la persecuzione degli Ebrei era, almeno in parte, l'effetto del quasi assoluto monopolio da essi realizzato nel mercato relativo ai prestiti ad interessi.

\section{Una difficile gestazione}

Il dibattito che si sviluppò nei primi anni del governo carolino intorno alla problematica degli ebrei testimonia un decisivo cambiamento di rotta, sia negli orientamenti intellettuali che nelle conseguenti scelte politico-giuridiche. La disamina preliminare della spinosa questione fu affidata, per espresso volere del Segretario di Stato, a Celestino Galiani: "autorità» giudicata capace di fornire il proprio parere «senza quelle preoccupazioni solite incontrate nelli spiriti ordinari, con mente libera, sciolta d'ogni sospetto $»^{53}$.

La scelta del monaco celestino, uomo di singolare talento che per quelle doti godeva di ampia fama ed era diventato, nel 1732, il Cappellano Maggiore del Regno, era molto significativa. Al di là delle implicazioni teologiche e religiose, il pronunciamento richiedeva, infatti, la forza di affermare un principio che era basilare per la tradizione giuridica meridionale: soltanto le leggi napoletane avevano il compito ed il potere di disciplinare la condizione degli ebrei nel Regno. La questione era sì teologica, ma andava discussa e decisa dal Sovrano, senza «veruna precedente licenza del Sommo Pontefice ${ }^{54}$.

La posizione, a dir poco coraggiosa, rispecchiava l'audacia dei riformatori, i quali godevano, sul piano politico, dell'appoggio del Segretario di Stato. II marchese, poi duca, di Salas, era un uomo colto e intraprendente, convinto sostenitore degli intellettuali d'indirizzo critico ed aperto a farsi interprete delle loro progettualità.

Confortati dal sostegno del potere politico, i novatores nutrivano la fiducia del

\footnotetext{
${ }^{52}$ Genovesi, 2005, p. 812.

${ }^{53}$ ASN, Ministero degli esteri, 4400, 9/2, Lettera di Montealegre a Contegna dell' 8 ottobre 1739.

${ }^{54}$ ASN, Ministero degli affari esteri, 4400, 9/4, Lettera di Pietro Contegna del 12 ottobre 1739 in risposta a Montealegre.
} 
cambiamento che sarebbe scaturito proprio dalla singolare sinergia con gli indirizzi di governo.

La triade, formata da Montealegre, Contegna e Galiani, è l'emblema della sintesi straordinaria che si realizzò tra cultura e potere politico nei primi anni del governo carolino. Nella fase di travaglio e di gestazione del provvedimento circa gli ebrei, le posizioni espresse dai due ecclesiastici, interpretate al governo dal Segretario di Stato, appaiono perfettamente allineate su una medesima linea coraggiosamente orientata verso l'innovazione.

L'apporto del Cappellano Maggiore appare il più incisivo. II singolare profilo della sua personalità lo rese protagonista indiscusso della contesa. Egli era, al contempo, un irreprensibile e stimatissimo ecclesiastico, ma anche un intellettuale d'indirizzo critico, acuto conoscitore della filosofia cartesiana e della scienza newtoniana: un religioso capace di concepire soluzioni ardite e di reale innovazione.

Il parere fornito dal Galiani confermò in pieno l'illuminata apertura del Cappellano Maggiore, che, senza mezzi termini, confermò che il Re potesse liberamente pronunciarsi sulla questione ebraica, senza dover chiedere alcuna licenza preliminare alla Corte di Roma. Il Cappellano si dichiarò favorevole all'ammissione nel Regno degli ebrei, i quali non avrebbero dovuto essere obbliati, né ad abitare in un ghetto, né ad indossare il segno. La plebe napoletatana, «insolente, rozza e superstiziosa", avrebbe tratto dall'uso del segno distintivo, l'occasione per attaccare i giudei e dar luogo a disordini e tumulti ${ }^{55}$.

La prospettiva eminentemente pragmatica del parere galianeo è evidente: la riforma doveva essere diretta a garantire quel pacifico stanziamento della comunità che sarebbe stato il presupposto degli auspicati vantaggi economici.

In questa prospettiva, Galiani si diceva convinto della necessità d'imporre agli ebrei il divieto di applicarsi agli studi giuridici addottorandosi in legge: per non tradire la ratio mercantilistica del progetto di riforma era necessario che gli interessi della comunità giudaica fossero indirizzati alla mercatura. Se essi fossero stati attratti dall'esercizio della lucrosa professione forense, avrebbero ingrossato le fila già fin troppo nutrite dei togati napoletani, sottraendo la loro energia alle attività più dinamiche e produttive.

Paladino della libertà di coscienza, Galiani, si diceva, inoltre, convinto che alcune tematiche tradizionalmente connesse alla questione giudaica, quali erano quelle relative all'esercizio della professione medica a favore dei malati cristiani, oppure al mestiere di balia, dovevano essere relegate all'arbitrio della coscienza e rimesse alla scelta del singolo fedele.

L'autorevolezza goduta dal Galiani fece sì che al suo parere si conformassero quelli resi dagli altri tre teologi che, sempre su incarico di Montealegre, furono scelti da Contegna per l'esame della questione. I tre religiosi indicati furono il cap-

${ }^{55} \mathrm{Cfr}$. Parere teologico sopra alcuni punti appartenenti all'introduzione degli Ebrei nei due regni di Napoli e Sicilia, in SNSP, ms. XXXI B-1, pp. 235-239. 
puccino Bernardo M. Giacco ${ }^{56}$, il benedettino Isidoro Sances, proveniente dalla Congregazione di Montecassino e già lettore di Morale nella Pubblica Reale Università di Napoli, e il francescano Bonaventura d'Arienzo. Essi erano ecclesiastici «liberi dalli popolari errori e pregiudizi», i quali nutrivano «sul proposito nostro, ottimi sentimenti» ed erano stati, per questi motivi, ritenuti capaci di «mettere in salvo la piissima coscienza del nostro Monarca, e stabilire la giusta e necessaria introduzione delli Giudei nelli Regni di Napoli e di Sicilia ${ }^{57}$.

È evidente che la scelta dei relatori contenesse già in sé la risposta ai quesiti. Se ci si fosse affidati all'ala conservatrice degli intellettuali e teologi napoletani, nulla sarebbe cambiato e i propositi di rinnovamento, ora adeguatamente garantiti dall'azione di governo montealegrina, sarebbero stati vanificati.

Il fronte dei riformatori doveva essere compatto nel concepire un piano in grado di incidere concretamente sulle condizioni materiali del Regno. Lontano da ogni preoccupazione religiosa, metafisica ed astratta, bisognava avere fiducia nelle nuove potenzialità vincendo ogni resistenza. Si delineava così quella visione preilluministica che, sul piano giuridico-politico, si inseriva appieno nella dialettica dei poteri d'Antico Regime e faceva emergere il divario tra i riformatori e coloro i quali restavano legati ad una visione statica dei ceti e dei blocchi di potere.

Ma proprio il travaglio imposto dal confronto con le resistenze feudali ed ecclesiastiche accrebbe nei riformatori la consapevolezza della necessità di indirizzare ogni sforzo verso la produttività finendo, quasi ottimisticamente, per far ritenere modificabili assetti parassitari che si erano incancreniti nelle strutture socio-istituzionali.

Innegabili erano, infatti, i condizionamenti che derivavano dal circolo vizioso entro cui era stretta l'economia regnicola. Le attività dello Stato erano quasi totalmente alienate a capitalisti privati. Per effetto di un meccanismo tipico dell' $A n$ cien Régime, la mancanza di tutela delle situazioni giuridiche patrimoniali scoraggiava qualsiasi altra forma di investimento che non fosse quella dell'acquisto delle rendite dello Stato e sconsigliava fortemente l'impiego di capitali in altri settori più rischiosi.

L'investimento nell'acquisto delle partite fiscali conveniva a tutti i possessori di liquidità ed era preferito da tutti i capitalisti, che, pertanto, si opponevano contro qualsiasi innovazione potenzialmente diretta a impedire il fruttuoso corso dei loro investimenti. Si creava così un fronte trasversale a difesa dello status quo.

La decisione sull'insediamento della comunità israelitica nel Regno toccava, dunque, più nodi problematici, avendo ricadute dirette sul piano economico, giuridico e politico-religioso.

Sotto quest'ultimo profilo, la questione ebraica offriva ai riformatori l'opportunità di demarcare il confine tra l'azione statale e le rivendicazioni della Chiesa.

\footnotetext{
${ }^{56}$ Su Giacco, cfr. Mastroianni, 1972.

${ }^{57}$ ASN, Ministero degli affari esteri, 4400, 9/4, Lettera di Pietro Contegna del 12 ottobre 1739 in risposta a Montealegre.
} 
Le pretese di controllo che, in modi più o meno palesi, provenivano da Roma costituivano una minaccia costante. Che il Regno fosse politicamente stretto in una vera e propria morsa, appariva evidente agli occhi di un acuto anticurialista qual era Pietro Contegna:

La Corte di Roma mette uno estremo terrore a tutte le persone ecclesiastiche di questo Regno [...] la medesima Corte esige dagli ecclesiastici napoletani non solamente che debbano difendere $\mathrm{o}$ a torto $\mathrm{o}$ a diritto tutte le sue pretensioni, ma anche vuole che cerchino con ogni immaginabile studio e sforzo di avanzare e di accrescere la potestà ecclesiastica sopra quella dei Principi; ed ha stabilito che sia instar sacrilegi il dubitare de potentia Summi Pontefici ${ }^{58}$.

La Chiesa napoletana era esecutrice delle volontà espresse, più o meno esplicitamente, da quella romana. Plausibile era, dunque, che le pressioni di Roma avrebbero facilmente vanificato qualsiasi progetto di rinnovamento. Anche gli esponenti più progressisti del clero napoletano apparivano succubi della curia romana e, all'atto pratico, apparivano incapaci di soluzioni originali.

Il costante controllo che da Roma era esercitato sulle manifestazioni di dissenso e di devianza religiosa costituiva, all'atto pratico, un'efficacissima arma di pressione politica. Le pretese egemoniche della Chiesa erano evidenti e si facevano strada, oltre che mediante il controllo capillare della vita civile, anche attraverso il persistente condizionamento degli esponenti ecclesiasticis9.

Proprio per tutelare la genuinità delle opinioni dei teologi prescelti per fornire il parere circa l'introduzione degli ebrei, Contegna implorava a vantaggio di questi ultimi la patente di teologi reale. Forte era il timore che costoro subissero ritorsioni in ragione delle opinioni espresse: una preoccupazione che dà il segno del clima complessivo in cui fu varato il provvedimento e dell'estrema difficoltà di partorire un provvedimento dalle molteplici implicazioni. Tuttavia, come ribadito dallo stesso Contegna nei toni di una vera e propria reprimenda nei confronti del governo, bisognava, rispetto alle pressioni della Corte di Roma, tenere «il piede fermo» e "pubblicare l'editto nelle forme che bisogna» ${ }^{60}$.

\section{A favore della «Nazione ebrea commerciante»}

II proclama, con cui il 7 febbraio 1740 si concesse alla Nazione ebrea il «Salvacondotto, perché possa venire a trafficare, ed a stabilire il Suo Domicilio nelli Regni delle due Sicilie, e loro dipendenze ${ }^{61}$, tradusse sul piano giuridico le aspirazioni dei riformatori. Anche se la ratio squisitamente economica impedisce di attribuire al provvedimento alcun significato di tipo egalitario o umanitario di più ampio

\footnotetext{
${ }^{58}$ Ibidem.

${ }^{59}$ Luogno, 2018, p. 312 e ss.

${ }^{60}$ ASN, Ministero degli affari esteri, 4401, 6/4, Lettera del Contegna del 30 gennaio 1740.

${ }^{61}$ Cfr. Prammatica VI, De expulsione Hebraeorum sive ludeorum, in Giustiniani, 1804, pp. 102-110.
} 
respiro, il contenuto di rottura che esso assunse rispetto alla passata tradizione è evidente ${ }^{62}$.

La carica innovativa del provvedimento risiede, innanzitutto, nell'affermazione di un'autorità politica che ammetteva e riconosceva giuridicamente uno spazio vitale per una comunità di infedeli, qual era quella israelita, in ragione della funzione economica che essa era chiamata a svolgere.

La riammissione degli ebrei rendeva palese, altresì, un sovvertimento dei valori di riferimento: lottando faticosamente contro pressioni secolari, l'approccio pragmatico, che aveva trovato terreno fertile tra le personalità più aggiornate, si faceva strada per concretizzarsi nell'azione di governo.

Nel dettaglio, le disposizioni erano finalizzate a creare condizioni di favore sia per gli ebrei che avessero deciso di stabilirsi definitivamente nel Regno, sia per quelli che avessero solo transitato entro i confini dello stesso: i primi avrebbero goduto di una condizione per molti versi addirittura più vantaggiosa rispetto a quella dei regnicoli, ai secondi sarebbero stati garantiti privilegi simili a quelli spettanti ai forestieri più favoriti.

«Grazie, privileggi, immunità, franchigie, esenzioni, e prerogative» erano concesse per la durata complessiva di cinquant'anni ed altri cinque di tolleranza. Nel loro insieme, esse erano funzionali allo scopo, apertamente dichiarato, di consentire ai regnicoli di prendere a modello la «Nazione ebrea commerciante», la quale "unicamente, e totalmente è intesa al Commercio» ${ }^{63}$. Gli ebrei avrebbero avuto

libera facoltà, e licenza, di venire, stare, trafficare, passare, o abitare colle loro famiglie, o senza esse; ed indi partire, tornare, e far negozio nelli nostri Regni, e Stati, senza verun impedimento, e molestia reale, o personale ${ }^{64}$.

E proprio l'ampiezza delle concessioni consente di giudicare il salvacondotto carolino, se confrontato con gli analoghi provvedimenti coevi, tra i più liberali ${ }^{65}$. Esso appare chiaramente ispirato, sia nella struttura che nei contenuti, alle Livornina, che costituì «un vero e proprio paradigma sulla cui falsariga diversi altri sovrani provarono a costruire analoghe concessioni, per quanto raramente con altrettanto successo" ${ }^{66}$. A Napoli, infatti, la breve vita del provvedimento e le vicissitudini legate alla sua applicazione ne depotenziarono enormemente l'efficacia.

\footnotetext{
${ }^{62}$ Di diverso avviso è Giura, secondo cui il provvedimento fu varato «perché, certamente, grazie al progresso degli "intelletti", molti pregiudizi, almeno tra le persone colte, erano caduti»: Giura, 1978, p. 4.

${ }^{63}$ Proclama, o vero Banno, con il quale si concede alla Nazione Ebrea un Salvacondotto, perché possa venire a trafficare, ed a stabilire il Suo Domicilio nelli Regni delle due Sicilie, e loro dipendenze, in Giustiniani, 1804, pp. 102-111.

${ }^{64}$ Ivi, p. 102.

${ }^{65}$ Ferorelli, 1915, pp. 245-248, Artom, 1950, pp. 23-31.

${ }^{66}$ Edigati, 2020, p. 944.
} 
L'evidente preoccupazione che gli ebrei non fossero attratti da un regno che non vantava una tradizione di tolleranza nei loro confronti, spinse il Sovrano a porre, in premessa all'editto, il riconoscimento di una fitta rete di immunità.

Giunti a Napoli, gli israeliti avrebbero goduto sia dell'immunità penale, per $\mathrm{i}$ reati già commessi in qualsiasi altro luogo, sia di quella civile, per i debiti contratti e rimasti insoluti prima dell'arrivo nel Regno. L'ampiezza delle garanzie promesse dal Sovrano si spingeva sino al punto di assicurare che

in verun modo Giudice alcuno, Foro, Tribunale, Magistrato ò Ministro per li suddetti pretesi debbiti, e delitti possano fare procedura, atti, o sentenza alcuna in pregiudizio delli suddetti Ebrei, e delle loro famiglie. [...] se mai per errore, o per altra causa, facessero il contrario, ogni loro atto, o procedura, e sentenza sia ipso iure nulla, e di niun vigore ${ }^{67}$.

Garantita da tali immunità, la comunità ebraica avrebbe potuto stabilirsi e vivere pacificamente nel Regno, fissandovi domicilio, esercitando liberamente la propria attività, senza dover per questo abiurare alla propria fede.

Un'ampia serie di concessioni assicurava agli ebrei la possibilità di osservare i precetti del proprio credo: il rispetto del sabato e degli altri giorni festivi ebraici e la possibilità di erigere dei cimiteri dedicati alla sepoltura dei loro estinti connotavano il riconoscimento di un ampio diritto di culto. In questa prospettiva, si inseriva anche la facoltà di apertura di un macello "per uso proprio" al fine di consentire la macellazione rituale delle carni «di ogni sorte di bestiame» ${ }^{68}$.

Si trattava di disposizioni importanti che, nel porre le condizioni per un insediamento stabile, pacifico e duraturo, riconoscevano agli ebrei un preciso spazio identitario. Al fondo, vi era la consapevolezza che nessuna duratura convivenza si sarebbe realizzata se la comunità insediatasi non fosse stata libera di conservare e coltivare la propria identità.

In tali favorevoli condizioni, gli ebrei avrebbero potuto esercitare le loro professioni e, tra queste, anche quella medica con la sola limitazione di assistere i cristiani con l'ausilio di un collega cristiano.

Peculiare attenzione era riservata alla tutela della capacità economica della comunità ebraica, cui era consentita la facoltà di esercitare il commercio di «mercatanzie d'ogni sorte». A questo scopo, le dotazioni personali, quelle strumentali all'esercizio del commercio e, in generale, le merci venivano riconosciute "franche, e libere da ogni pagamento di gabella, di Dogana, e di passo" sia all'ingresso che all'uscita dal Regno ${ }^{69}$. A corollario di tale impianto normativo, alcuni magazzini, nella Dogana di Napoli e nelle altre presenti nel Regno, erano, liberamente e senza obbligo di pagamento alcuno, posti a disposizione dei mercanti ebrei.

Dal punto di vista giuridico-commerciale, molto rilevante era il riconoscimento

\footnotetext{
${ }^{67}$ Proclama, Giustiniani, 1804, pp. 102-111.

${ }^{68}$ Ivi, p. 108.

${ }^{69}$ Ivi, p. 109.
} 
di «piena ed ampia fede» che era riservato ai libri di commercio dei mercanti ebrei, che, "se tenuti secondo l'ordine" dovevano essere equiparati a quelli dei mercanti regnicoli ${ }^{70}$. La piena ed ampia fides riconosciuta ai giudei suggellava sul piano giuridico una straordinaria conquista: il binomio, buona fede - infedele, non doveva essere considerato un ossimoro.

Diretta conseguenza di questa nuova visione era il riconoscimento in capo agli israeliti del diritto di fare testamento e di stipulare liberamente contratti «ordinari e minuti» senza autentica notarile o assistenza di testimoni.

A spezzare il ben noto circolo vizioso che legava il divieto per gli ebrei di possedere beni stabili all'esercizio delle usure, che infatti restavano vietate nei confini del Regno, interveniva poi la norma che ammetteva espressamente la capacità degli ebrei di comperare «beni stabili», ad eccezione dei "Feudi, o altra specie di diritti, e di beni, per li quali abbiano autorità e giurisdizione sulli Cristiani $»^{71}$.

Il riconoscimento di uno spazio giuridico per l'acquisto dei beni stabili costituiva una radicale innovazione a vantaggio di una comunità che aveva dovuto far ricorso a formule giuridiche alternative, quali per esempio lo jus kazakà, per aggirare il divieto ${ }^{72}$.

Inoltre, era innegabile che proprio quella proibizione, fortemente limitativa della capacità d'agire degli ebrei, avesse finito per legittimare l'esercizio dell'usura, e per questa strada, aveva alimentato quel diffuso sentimento di odio e di pregiudizio che aveva condizionato i rapporti tra giudei e cristiani.

A completare il quadro normativo d'indiscutibile favor per gli ebrei, intervenivano, poi, le norme che, dal punto di vista giurisdizionale, sottraevano le controversie insorte tra ebrei e cristiani alla macchina giudiziaria ordinaria e le attribuivano alla cognizione del Delegato. Scelto tra i membri del Supremo Magistrato del Commercio, il Delegato avrebbe giudicato le liti insorte tra giudei e quelle nate tra questi ultimi e i cristiani. A tale scopo sarebbero stati nominati tre Delegati: uno a Napoli e gli altri due a Palermo e a Messina. Più precisamente, l'editto disponeva che ai Delegati sarebbe spettata la cognizione di

tutte le cause, che sorgeranno frà un Cristiano, ed un Ebreo, e tutte quelle Cause, che si agiteranno fra Ebreo, ed Ebreo purché si tratti di qualche delitto, che meriti pena maggiore della relegazione, o dell'esilio: Imperciochè a riguardo di ogni altro genere di cause che dovranno agitarsi, frà Ebreo, ed Ebreo, vogliamo, che siano Giudici privatamente i loro Massari, dalli quali se mai si sentiranno gravati, e venga loro usata violenza, sia ad essi Ebrei lecito così ne' casi sudetti, come in qualunque altro di manifesta, e grave oppressione di ricorrere alla nostra Real protezione ${ }^{73}$.

\footnotetext{
70 Ivi, p. 107.

${ }^{71}$ Ivi, p. 108.

${ }^{72}$ Cfr. i recenti studi di Andreoni-Gasperoni, 2018, pp. 9-18; Graziani Secchieri, 2018, pp. 19-48; Andreoni, 2018, pp. 49-72.

${ }^{73}$ Proclama, Giustiniani, 1804, p. 105.
} 
In questa prospettiva, la maggioranza delle controversie in cui fossero stati coinvolti gli appartenenti alla comunità ebraica si sarebbe consumata in una dimensione privatistica indipendente dallo Stato, secondo il rito e le tradizioni proprie del mondo giudaico ${ }^{74}$. L'autonomia, seppur riconosciuta per i soli affari giurisdizionali di minore rilevanza, garantiva alla comunità ebraica la possibilità di conservare la propria identità e di escludere l'eteronomia dell'intervento statale.

D’altro canto, anche per le cause più rilevanti, l'attribuzione della funzione giurisdizionale ai soli Delegati finiva per sottrarre gli ebrei alla macchina giudiziaria tradizionale, addirittura anche in grado d'appello. Le impugnazioni delle pronunce, rese in primo grado dai Delegati, sarebbe, infatti, avvenuta innanzi al neoistituito Supremo Magistrato del Commercio.

Articolata in tal modo, la norma collegava testualmente il programma di riammissione degli ebrei all'introduzione del nuovo organismo di giustizia commerciale, dimostrando quanto fosse forte la preoccupazione che anche l'intervento della giustizia ordinaria potesse impedire, nei fatti, la realizzazione dell'auspicato piano di ammodernamento.

\section{Nuovi mercanti e antichi baroni}

Incidendo profondamente sugli assetti economici e sociali da tempo consolidati$\mathrm{si}$, il proclama suscitò, dentro e fuori i confini del Regno, una forte reazione, che si alimentò, ad un livello più subdolo, ma non per questo meno capace di condizionare il piano dei rapporti espliciti, di un humus fatto di credenze, pregiudizi, antichi livori. II complesso di questi fattori, passionali ed istintivi, spingeva per emergere ed influenzare il versante palese dei rapporti giuridici: una dinamica che era favorita da coloro che, proprio facendo leva su quel diffuso sentimento di ostilità, lo strumentalizzavano in funzione degli interessi di parte.

Utilizzando quei moventi, in molti agirono, da più parti, nel tentativo di indurre la Corona a compiere un passo indietro rispetto alle posizioni assunte nell'editto. A Napoli, nello stesso anno della pubblicazione del bando, fu diffuso un pamphlet dal titolo «Sentimento intorno al Proclama, o sia Bando, col quale s'introducono gli ebrei nelli Regni delle Due Sicilie, e loro dipendenze, pubblicato in Napoli li 3 febbraio in quest'anno $1740 »^{75}$. Lo scritto conteneva un durissimo attacco contro gli ebrei e riassumeva la posizione della curia napoletana che era fedele esecutrice di quella romana.

\footnotetext{
${ }^{74}$ Sulla giurisdizione dei Massari, che era la massima autorità civile della comunità ed era chiamata a giudicare secondo la legge ebraica ed i suoi principi, cfr. Toaff, 1965, pp. 275-276. L'editto carolino prevedeva che, con l'arrivo delle prime quaranta famiglia nelle città di Napoli, Palermo e Messina e delle prime venti nelle altre, sarebbe stato nominato il consiglio incaricato di eleggere i Massari. All'autorità di questi ultimi sarebbe spettato anche il compito di decidere dell'eredità lasciate dagli ebrei ab intestato. Cfr. Proclama, in Giustiniani, 1804, p. 111.

${ }^{75}$ Ferorelli, 1915, pp. 252-253.
} 
Iniziò, inoltre, a circolare ad arte la voce che l'editto fosse il frutto delle negoziazioni di Francesco Ventura, che era stato indotto a trattare l'affare della riammissione in cambio di una "grossa somma di denaro accordatagli dal Corpo degli Ebrei, cacciati da Bordeau» ${ }^{76}$.

La «ridicola comedia», alimentata da bugie «inventate per isciocchezza, e per mancamento di veraci notizie» coinvolse, anche il Sovrano, su cui alitava il sospetto di una trattativa con la Nazione ebrea: in cambio dell'accoglienza, la comunità israelita avrebbe "somministrato al Re Nostro Signore un milione di scudi» ${ }^{77}$.

L'insieme di queste voci, che si erano nutrite dell'insofferenza della curia romana $^{78}$, era finalizzata a creare intorno all'editto un generale clima di diffidenza: lungi dal perseguire i proclamati obiettivi di produttività, esso veniva screditato quale frutto di un circolo di corruzione di cui erano protagonisti i più stimati uomini di governo e, addirittura, la persona stessa del Sovrano.

Per motivazioni tra loro diverse ma certamente convergenti, quell'onda montante di «disseminazioni e mormorazioni» era, dunque, incessantemente alimentata.

I primi a dirsi convinti dell'inutilità del provvedimento erano i mercanti, i quali addirittura si dimostravano "offesi dal Proclama», nella misura in cui il Re aveva definito la Nazione Ebrea come quella «unicamente» versata negli affari ${ }^{79}$. I giudei, anzi, sarebbero stati la «rovina dei mercanti cristiani», i quali, avrebbero sofferto gli effetti di una concorrenza sleale praticata a basso prezzo e con merci di scadente qualità ${ }^{80}$. Mentre, dunque, l'arrivo degli Ebrei era guardato dai riformatori come un'iniezione di abilità finanziaria, necessaria al tessuto napoletano, i sudditi mercanti additavano la novità come una minaccia.

Accanto all'opposizione dei mercanti, la riforma suscitava la comprensibile reazione dei nobili. Essi, «avezzi a vivere senza industria alcuna ed oziosi, sostenendosi colle fruttuose rendite degli Arrendamenti, e delli Fiscali», si erano apertamente schierati «contro la venuta degli Ebrei in questo Regno», celando «sotto

\footnotetext{
${ }^{76}$ Le citazioni sono tratte da Osservazioni su di una scrittura presentata a V. E. e intitolata Riflessioni che anno dato e tuttavia danno motivo alli generali discorsi sopra l'erezione del nuovo tribunale di commercio ed in seguito sul Proclama ossia Salva condotto, che si è conceduto alla nazione ebrea, conservato in ASN, Ministero degli affari esteri, 4401, 5, pubblicata in appendice a Natale, 2014, p. 333-347. La citazione è a pp. 342-343.

77 Ivi, p. 343.

78 Una sicura testimonianza in tal senso è nelle Riflessioni su la lettera venuta di Roma in proposito degli ebrei, in ASN, Ministero degli affari esteri, 4401, 6/9. Anche a Roma si era diffusa «la diceria che S.M. per godere alcuni millioni offertigli dagli ebrei abbia risoluto introdurli in questo Regno».

${ }^{79} \mathrm{Cfr}$. Riflessioni che anno dato e tuttavia danno motivo alli generali discorsi sopra l'erezione del nuovo tribunale del commercio ed in seguito sul Proclama ossia Salva condotto, che si concede alla nazione ebrea, conservato in ASN, Ministero degli affari esteri, 4401, 5, pubblicato in appendice a Natale, 2014, p. 332.

${ }^{80}$ Ivi, p. 332.
} 
la maschera dello zelo della Religione Cristiana», la difesa del proprio interesse ${ }^{81}$. II nucleo della critica proveniente dal ceto baronale era espressivo di quella logica di blocco che, di fatto, impedì alle iniziative, pur avviate nella prima fase del governo carolino, di potersi realizzare.

La fiera ostilità con cui i baroni guardavano alle disposizioni a favore degli ebrei svelava, come una cartina al tornasole, la capacità insita nella riforma di intaccare il nocciolo duro di cui si sostanziava l'assetto parassitario dell'economia regnicola.

L'impossibilità di trovare nell'apparato statale un'adeguata tutela delle situazioni patrimoniali, infatti, aveva indotto i benestanti ad assumere un orientamento per così dire timido, di cautela rinunciataria, nella conduzione dei propri investimenti, ed a preferire per essi una strada più comoda e più sicura rispetto all'impiego in attività più rischiose. In tal modo, il movimento dei capitali privati era stato da tempo indirizzato verso l'acquisto delle rendite fiscali.

L'opzione a favore di questa tipologia d'investimento alimentava nel Regno una spirale negativa, poiché enormi flussi di danaro erano sottratti al traffico produttivo con effetti a catena che avvantaggiavano i benestanti, ma finivano per ripercuotersi sugli strati più deboli della popolazione costretti a vivere ai margini ${ }^{82}$. L'economia reale viveva in una condizione di tutela inerte dominata dal circolo vizioso parassitario goduto dall'establishment.

II blocco che la vendita degli uffici e delle imposizioni tributarie aveva determinato nel meccanismo pubblico non poteva essere rimosso. La condizione di paralisi derivava, in primo luogo, dall'impossibilità di abolire dazi, gabelle, diritti, poiché i relativi gettiti erano stati alienati a privati, che avevano versato alla Corte anticipatamente il corrispondente capitale.

D'altro canto per il governo vi era la necessità di tener fede alle rendite promesse e garantirsi la possibilità di trovare i capitali continuamente necessari alla pubblica gestione. La paralisi economica era divenuta una condizione stabile ed irreversibile.

Rispetto a tale stato di fatto, il provvedimento di riammissione degli ebrei faceva paventare delle pericolose inversioni di tendenza, dimostrando un interesse per la produttività che si poneva in antitesi con l'impiego parassitario del denaro.

Sotto il profilo giurisdizionale, inoltre, appare molto significativo che la nobiltà paventasse anche un effetto ulteriore della riammissione degli ebrei. Inserendosi appieno nella dialettica cetuale, l'iniziativa era colta come un primo passo per realizzare

quello che si dice: cioè che il Governo voglia fare intendere alli Sudditi dei Baroni, che tutti quelli che pagheranno cento scudi potranno esentarsi dai Tribunali dei Feudatari, e ricorrere ai Tribunali Regi per farsi fare giustizia ${ }^{83}$.

\footnotetext{
${ }^{81}$ Ivi, p. 343.

${ }^{82}$ Il complesso ed intricato meccanismo economico emerge nelle diagnosi dei componenti della Giunta borbonica di Commercio. Sul punto cfr. I'ampio materiale documentario in SNSP, ms. XXI d 30.

${ }^{83}$ Riflessioni, in Natale, 2014, p. 332.
} 
A pochi mesi dal varo del Supremo Magistrato del Commercio, il timore dei nobili di soffrire ulteriori sottrazioni di potere giurisdizionale era certamente giustificato. Le iniziative assunte dal governo montealegrino andavano in un'unica direzione che era, al contempo, anticuriale ed antibaronale. Appariva, dunque, chiaro il senso di una strategia politica finalizzata a porre in discussione le fondamenta della società cetuale d'Antico Regime: un progetto ambizioso che mirava a «disincagliare» ${ }^{84}$ il blocco dei poteri attraverso interventi plurimi, ma univocamente diretti ad innestare moventi di modernizzazione.

\section{Riformatori a confronto: tra audacia e prudenza}

Il provvedimento di riammissione degli ebrei aveva suscitato, da più parti, una reazione di ostilità che, se da un lato offriva un'indiretta conferma della validità dell'iniziativa riformistica, d'altro canto induceva ad una riflessione, oltre che sul contenuto, anche sui modi e i tempi dell'iniziativa. All'indomani dell'adozione dell'editto, gli orientamenti di coloro che pure ne erano stati fautori finirono, così, per differenziarsi.

L'atteggiamento, più libero e al contempo più intransigente, continuò ad essere assunto da Pietro Contegna. Parole roventi erano quelle che l'abate di Arienzo indirizzava a "monaci, frati», diventati protagonisti di una "ridicola comedia» costruita ad arte con "favole e menzogne». Animati da «farisaico zelo e [...] da altri irragionevoli passioni, intenti e capricci», gli ecclesiastici istigavano i napoletani contro i giudei, accusati di essere "furfanti ed ingannatori»"

Paladino indiscusso della causa ebraica, Contegna si diceva convinto che nessun ghetto dovesse sorgere nel Regno e nessun segno distintivo dovesse essere imposto. Chiara era la finalità di una così larga apertura: creare le condizioni per un insediamento stabile e pacifico, garantito dall'autorità politica contro gli attacchi degli ecclesiastici più retrivi e della «minuta plebe», rea di «mille beffe e derisioni».

Per questa strada, il proclama, di fatto estraneo a qualsivoglia scopo umanitario ed egualitario, diventava, giocoforza, lo strumento di promozione di una nuova e singolare modalità di convivenza tra cristiani e infedeli. Un approccio pragmatico, libero dai condizionamenti derivanti da stereotipi e pregiudizi, si faceva, faticosamente, strada.

${ }^{84}$ D'Ippolito, 2006.

${ }^{85}$ La citazione è tratta da Osservazioni su di una scrittura presentata a V. E. e intitolata Riflessioni che anno dato e tuttavia danno motivo alli generali discorsi sopra l'erezione del nuovo tribunale di commercio ed in seguito sul Proclama ossia Salva condotto, che si è conceduto alla nazione ebrea, conservato in ASN, Ministero degli affari esteri, 4401, 5, in appendice a Natale, 2014, pp. 333-347. II sentimento di diffidenza nei confronti degli ebrei era stato favorito da «un'agguerrita predicazione antiebraica dei Francescani, che additava i giudei come corruttori della società, sia dal punto di vista morale che economico»: cfr. Dani, 2021, p. 67. 
A riguardo, è molto significativo che proprio Contegna non nascondesse che le sue idee erano d'indirizzo contrario all'opinione di molti «infelici Statisti e Politi$\mathrm{ci})^{86}$.

Ogni questione relativa agli ebrei alimentava un dibattito per sua natura divisivo che, oltre a vedere contrapposte le opinioni tra veteres e novatores, evidenziò presto l'esistenza di sensibili differenze anche in seno agli stessi riformatori: una distanza che divenne evidente allorquando le pressioni per la revoca, o almeno per la modifica, del proclama divennero esplicite.

Ben prima che la corte carolina manifestasse orientamenti di governo a dir poco più prudenti, divenne sempre più chiara l'assenza sulla questione di un'intesa di fondo tra i riformatori. La mancanza di un generale consenso si rivelò in termini espliciti allorquando si trattò di difendere il provvedimento contro la richiesta di una sua revoca.

Il complesso dei valori coinvolti nella disputa imponeva di compiere scelte inesorabili, di sacrificare alcuni interessi a vantaggio di altri. L'impresa di realizzare questo difficile bilanciamento rese visibile la frattura tra chi era propenso al cambiamento radicale e chi manifestava la prudenza del possibile.

Una questione di sicura rilevanza che, come tale, incise fortemente sulla diversità degli orientamenti, risiedeva nei rapporti con la Santa Sede. Sul presupposto di dover salvare la «vera fede», infatti, il tentativo d'ingerenza della curia romana nelle scelte del governo di Napoli era continuo. Già all'indomani della pubblicazione del proclama di riammissione, da Roma ne era stata richiesta, più o meno esplicitamente, la revoca o, quanto meno, la sostanziale revisione.

Lo scontro, ammantato di preoccupazioni religiose, era in realtà politico ed economico ed aveva immediate ricadute sul piano giuridico. Non sfuggiva alla Santa Sede che l'introduzione degli ebrei fosse ritenuta una misura indispensabile nel più ampio processo di ammodernamento socio-istituzionale del Regno: era evidente che proprio questa nuova proiezione accrescesse i motivi dell'insofferenza romana.

Le pesanti accuse al governo carolino, responsabile dell'insano progetto di riammissione, erano state raccolte in "due scritture della Romana Inquisizione intorno all'introduzione degli ebrei nelli Regni di Napoli e di Sicilia» ${ }^{87}$. Roma chiedeva, in buona sostanza, di revocare i più importanti benefici che erano stati concessi agli israeliti: attraverso lo smantellamento, progressivo e inesorabile, della rete di protezione creata a favore della comunità ebraica, il provvedimento sarebbe stato reso vano.

\footnotetext{
${ }^{86}$ Ivi, p. 344.

${ }^{87}$ Nella prima delle due scritture era proprio contenuta la «censura formata dalla Congregazione del Santo Officio sulla deliberazione di S. M. di introdurre gli ebrei nelli due Regni di Napoli e di Sicilia». Cfr. Riflessioni sulle due scritture della Romana Inquisizione intorno all'introduzione degli ebrei nelli Regni di Napoli e di Sicilia, in ASN, Ministero degli affari esteri, 4401, f. 6/5.
} 
Qualsiasi limitazione dei privilegi e dei benefici avrebbe depotenziato l'efficacia del provvedimento, giacché era

manifesto che se agli ebrei si negano rigorosamente li sudetti punti ed indulti, certamente è impossibile che possano stare nelli Regni di Napoli e di Sicilia senza vivere fra infinite angustie, tra pazzie, molestie e preoccupazioni; si che vengono ad essere costretti o a non pensare di fissare la lor dimora nelli suddetti Regni ${ }^{88}$.

In queste condizioni, a Napoli sarebbero giunti soltanto gli «ebrei miserabilissimi privi di ricchezze e di commodità»: in questo modo, il provvedimento carolino avrebbe perso ogni significato.

Evidente era, dunque, che le pressioni della Santa Sede svelassero il malcelato tentativo di controllare la politica regnicola. Contro questa pretesa, si schierava audacemente Pietro Contegna che motivava la propria posizione giurisdizionalista utilizzando gli strumenti dell'efficace lezione storica: era

noto ad ogni uno il quale tiene mezzana cognizione della Storia di questi Regni che la Romana Corte abbia sempre preteso ed ambito di ottenere non solamente il dominio diretto che conserva in Napoli con l'uso dell'Investitura ma anche il dominio effettivo ed utile ${ }^{89}$.

Il progetto di riammettere gli ebrei aveva reso palese la tradizionale volontà di controllo della corte di Roma, la quale pretendeva di realizzare su Napoli quanto era riuscita ad ottenere da tutti «i piccioli Principi», i quali «riconoscevano l'alto dominio del Papa». Per questo motivo, i Papi attuavano una politica volta a far sì che

li Re di Napoli e di Sicilia sieno in questo Regno poveri e bassi, che li loro sudditi siano malcontenti e malmenati, che gli ecclesiastici vi godano una somma autorità potenza e ricchezza, e siano affatto liberi da qualunque giurisdizione ed autorità delli loro Re; perché con tal mezzo se il Re di Napoli e di Sicilia non possiede altri Regni in tal caso essendo povero e di poca autorità e tenendo li suoi Popoli miserabili e non avendo autorità se non sopra una parte di loro può facilmente sperare la Corte di Roma che venga un giorno la tanta sospirata occasione di cacciarlo interamente dal Regno ${ }^{90}$.

A ragione, pertanto, bisognava confermare con fierezza quanto il governo napoletano aveva stabilito. Ancor prima della comunità ebraica, era necessario difendere la valenza giuridica dell'editto di riammissione: quel provvedimento assumeva un significato ulteriore e ben più rilevante. Se era vero che «la Corte di Roma cerca(va) ogni occasione di intromettersi nel governo degli altri Stati ${ }^{91}$,

\footnotetext{
${ }^{88}$ Ibidem.

${ }^{89}$ Ibidem.

${ }^{90}$ Ibidem.

91 Ibidem.
} 
bisognava statuire l'autonomia decisionale del Sovrano contro ogni altra pretesa. Soltanto l'affermazione di tale indipendenza avrebbe spezzato il circolo vizioso di subalternità e sottosviluppo che caratterizzava la condizione del Regno.

D'altro canto, la contraddizione in cui incorreva la Santa Sede era manifesta: se fossero stati credibili ed attendibili i motivi religiosi sostenuti per negare l'accoglienza degli ebrei, questi ultimi avrebbero dovuto essere espulsi prima di tutto dai possedimenti della Chiesa. Agli occhi dei più avveduti, recava meraviglia che da la curia romana sollecitasse un intervento normativo che proibisse a Napoli quanto era, dalla Chiesa stessa, consentito nei territori ad essa direttamente sottoposti. La manovra in atto, chiaramente politica ed economica, era apertamente denunciata dal Delegato:

È paruto assai strano, e per verità ha recato meraviglia che nella città di Roma ove da tanti secoli sono stati dalli Sommi Pontefici ricevuti, e trattenuti gli ebrei, siasi poco bene intesa la risoluzione presa da S. M. di permettere alli medesimi il domicilio nelli suoi Stati e Regni ${ }^{92}$.

A sostegno delle posizioni assunte dal Sovrano, s'invocava, dunque,

l'esemplo del passato prudentissimo Sommo Pontefice, il quale perché voleva mettere in piedi un florido commercio nella città di Ancona opportunissima a tale effetto, stimò principalmente necessario di raccogliere nella suddetta città il maggior numero di ebrei che fusse possibile ${ }^{93}$.

Suffragato da tali modelli, il regno di Napoli doveva rivendicare con forza la legittimità dell'intervento normativo. Anche «nell'Orbe Cattolico» dovevano distinguersi «assai esattamente gli interessi della Religione da quelli dello Stato» ${ }^{94}$.

Da tale considerazione, che riassumeva una posizione tipica del giurisdizionalismo, discendeva, quale naturale corollario, l'affermazione secondo cui spettasse unicamente al Sovrano di decidere circa l'introduzione degli ebrei: di «questo suo giudizio il quale appartiene al governo temporale de' Popoli non è tenuto a renderne conto se non a Dio»" ${ }^{95}$.

Secondo Contegna, infatti, l'autorità sovrana, ispirata ed approvata da Dio, era l'unica ad essere capace di anteporre il bene comune all'interesse di parte. E l'interesse generale andava protetto, senza deroga alcuna, contro gli interessi

\footnotetext{
${ }^{92}$ Riflessioni su la lettera venuta di Roma in proposito degli ebrei, in ASN, Ministero degli affari esteri 4401, 6/9.

${ }_{93}$ Ibidem. Al di là degli avvenimenti particolari, come la persecuzione dei marrani (su cui cfr. Rosenberg, 1935, pp. 306-323), la Santa Sede aveva elargito al gruppo levantino di Ancona un trattamento particolare e più favorevole anche in confronto a quello della comunità di Roma, soprattutto in campo economico, si cfr. Simonsohn, 2017, p. 217. Sul tema, di recente, Andreoni, 2019.

${ }^{94}$ Riflessioni sulle due scritture della Romana Inquisizione intorno all'introduzione degli ebrei nelli Regni di Napoli e di Sicilia, in ASN, Ministero degli affari esteri, 4401, 6/5. ${ }^{95}$ Ibidem.
} 
particolaristici96.

Tuttavia, entro i confini del Regno, il clima di tensione creatosi intorno alla riammissione dei giudei imponeva ulteriori motivi di riflessione: frequenti erano gli episodi d'intolleranza e di violenza che si consumavano a danno degli Israeli$\mathrm{ti}^{97}$. Complice anche la ritardata liquefazione del sangue di San Gennaro che, il 5 maggio 1742, era stata imputata alla presenza di un ebreo nella folla dei fedeli cristiani, una nuova ed accurata disamina degli articoli del proclama apparve ben presto indispensabile.

Appena dodici giorni dopo l'infausto evento da cui erano scaturiti disordini che erano sconfinati in violenza, il 17 maggio 1742, Pietro Contegna, Celestino Galiani e Bernardo Tanucci $^{98}$ venivano insigniti del compito di un nuovo pronunciamento sulla questione ebraica.

Più nel dettaglio, la missione affidata alla triade era di procedere all'esame delle critiche sollevate dall'Arcivescovo di Napoli Giuseppe Spinelli, il quale aveva suggerito alcune modifiche da apportare al proclama reale per renderlo conforme alla volontà della curia romana ${ }^{99}$. La posizione di Spinelli, da lui stesso riassunta in un Foglio denso di osservazioni, era espressiva della chiara volontà di annichilire gli effetti del salvacondotto.

Sebbene il Cardinale non richiedesse la formale revoca del proclama, l'insieme delle sue richieste agiva, sul piano sostanziale, nella medesima direzione. In questa prospettiva, si collocava la richiesta di abolizione delle immunità, la proposta di introdurre l'obbligo per gli ebrei di indossare il segno, l'istituzione del ghetto, la disciplina di limiti all'acquisto di beni immobili, eccezion fatta per la sola abitazione all'interno dell'edificando ghetto.

La disamina di Spinelli riguardava, in buona sostanza, tutti i punti fondamentali della contesa, su cui ampio era stato il confronto nella fase di preparazione dell'editto.

La richiesta che su quelle questioni vi fosse un riesame rendeva chiara la svolta in atto: significava ammettere l'eventualità di un arretramento rispetto alle posizioni espresse nel proclama. E non v'era dubbio alcuno che, ove fosse avvenuta, la riforma avrebbe reso palese la volontà del Sovrano di conformarsi ai dettami della curia.

\footnotetext{
${ }^{96}$ Luongo, 2018, p. 385.

${ }^{97}$ Ajello, 1976, p. 673.

${ }^{98}$ Sui suoi rapporti con Montealegre e Santisteban: Ascione, 2000, pp. 45-48. Su Tanucci, cfr. Luongo2008a, p. 138 e Luongo 2008b, pp. 999-1015.

${ }^{99}$ Le posizioni di Spinelli furono da questi riassunte nel Foglio del Signor Cardinale Arcivescovo contenente alcune osservazioni sopra il proclama o sia banno, col quale fu permesso agli ebrei di venire a traficare nel regno delle Due Sicilie con istabilirvi il loro domicilio, in ASN, Ministero degli affari esteri, f. 4402. Su di esso si cfr. anche Giura, 1978, p. 65. Qualche anno dopo fu proprio lo stesso Cardinale Spinelli che tentò d'introdurre l'Inquisizione a Napoli suscitando una vera e propria rivolta. L'evento screditò definitivamente Spinelli agli occhi del Sovrano.
} 
Per questo motivo, Contegna manifestò di voler tenere salde le proprie posizioni facendo appello alla «maturissima» riflessione che già aveva preceduto l'emanazione del salvacondotto.

L'intransigenza del suo punto di osservazione offre conferma della tenacia con cui egli sostenne l'iniziativa riformistica; ma, l'ostinata resistenza che aveva indotto, fino a quel momento, a respingere al mittente ogni tentativo di ingerenza ecclesiastica non sembrava più agire con ugual forza in coloro che pur erano stati paladini della causa ebraica. Più moderato e prudente fu, infatti, l'atteggiamento assunto da Celestino Galiani, il quale si spinse sino al punto di giudicare «eque e ragionevolissime» le richieste del Cardinale Spinelli. E proprio in accordo con l'alto prelato, si disse favorevole ad introdurre una limitazione circa i luoghi del Regno dove gli ebrei avrebbero potuto insediarsi: sarebbe stato utile privilegiare il loro stanziamento nei luoghi di mare dove avrebbero potuto più facilmente commerciare.

Prendendo le distanze dalle posizioni da lui stesso espresse poco tempo prima, Galiani ammetteva anche la possibilità di costituire un ghetto e, in attesa di identificare il luogo più idoneo allo scopo, di introdurre l'obbligo per gli ebrei di vivere in abitazioni loro riservate senza potersi mescolare con i cristiani. Eccezion fatta per l'obbligo di indossare il segno, sui cui ferma restava l'opposizione galianea, per il resto il parere si conformava ai voleri dell'autorità ecclesiastica.

In questa prospettiva, in cui sembrava realizzarsi il perfetto e prudente adeguamento alle pretese della Chiesa, resisteva invece la volontà del Galiani di difendere l'autonomia dell'autorità sovrana. Le modifiche dovevano, infatti, essere apportate sotto la veste di «spieghe», ossia di chiarimenti alle precedenti statuizioni del Re, che, almeno in linea di principio, non dovevano essere smentite ${ }^{100}$.

Seppur sotto la falsa veste di una mera interpretazione autentica, la dichiarazione galianea di acconsentire alle modifiche testimoniava un nuovo orientamento di prudenza, che appariva motivato dalla necessità di realizzare la mediazione tra opposte tesi.

Era il primo segnale di una svolta che, manifestandosi nelle posizioni di alcuni intellettuali, dava il segno del cambiamento in atto nella politica carolina e nei rapporti con Roma e che risentiva, altresì, degli effetti del Concordato raggiunto con la Santa Sede: il complesso di questi fattori induceva a limitare le pretese.

II consenso intorno ad una linea di cautela trovava d'accordo anche Bernardo Tanucci. Da toscano, abituato a vedere i benefici effetti dell'accoglienza degli ebrei, egli dichiarava di trovarsi in difficoltà a concepire delle limitazioni per la comunità ebraica. Ciò nondimeno lo stesso Tanucci, facendo proprie le ragioni della mediazione, riteneva condivisibile la scelta di aderire alle richieste di Spinelli, eccezion fatta per l'obbligo d'indossare il segno, su cui, concordando con Galiani, manteneva il proprio contrario proposito ${ }^{101}$.

\footnotetext{
${ }^{100}$ ASN, Ministero degli affari esteri, 4402, Galiani a Montealegre, 1 giugno 1742.

${ }^{101}$ ASN, Ministero degli affari esteri, 4402, Tanucci al Re, 11 giugno 1742.
} 


\section{Parabole parallele}

La bozza del nuovo editto che, formalmente, era chiamato a spiegare il vecchio, ma che di fatto doveva recepire le richieste formulate dal Cardinale Spinelli, era affidata al Contegna, che, dal canto suo, continuava a manifestare una decisa insofferenza verso la linea compromissoria. Era innegabile che l'iniziativa riformistica stesse avviandosi a percorrere una fase discendente, espressiva del prevalere di un nuovo orientamento più cauto, moderato e prudente.

Si trattava di un dato di fatto che non sfuggiva ai contemporanei e, primo tra tutti, non sfuggì a Francesco Ventura, che, chiamato a fornire il proprio parere, si manifestò apertamente contrario ad un pronunciamento mediante un nuovo formale editto, suggerendo che le modifiche fossero accolte solo sotto forma di "segreto biglietto» da inviare al Delegato della Nazione ebrea.

La presa di posizione del Ventura era tutt'altro che formalistica: si trattava di tutelare la legittimità del potere sovrano. Gli aggiustamenti, resisi evidentemente necessari a tutela dei rapporti con Roma, dovevano riguardare il piano pratico, non quello formale. Inoltre, su almeno un punto, il governo non avrebbe dovuto far alcun passo indietro: gli ebrei non avrebbero indossato alcun segno distintivo. Qualsiasi emendamento fosse stato apportato in tal senso avrebbe smentito e screditato il Sovrano, che aveva promesso alla comunità israelita un pacifico insediamento. Le «modifiche» del proclama sarebbero state, inoltre, comunicate segretamente all'Arcivescovo di Napoli ed al Delegato della Nazione ebrea, cui sarebbe spettato il compito di darne effettiva esecuzione.

Ed infatti, con tali accorgimenti, il complesso negoziato si concluse con l'espressa accettazione da parte della curia romana il 19 febbraio 1743. L'epilogo della vicenda, peraltro non definitivo, era il chiaro segno del cambiamento in corso: una nuova linea di moderazione era prevalsa contro l'ostinata difesa delle posizioni più ardite di cui si era fatto portavoce il Contegna, al riguardo significativamente soprannominato «pontefice degli ebrei» ${ }^{102}$.

II ruolo centrale assunto dall'abate di Arienzo nel corso dell'intera vicenda emerge indirettamente da quanto accadde successivamente alla sua morte nel luglio del 1745, allorquando, infatti, la situazione precipitò definitivamente. L'onda d'insofferenza nei confronti dell'audace iniziativa che il giovane Re aveva assunto, su iniziativa del Montealegre, montò sempre più minacciosa e finì per travolgere la persona del Sovrano, già soprannominata Carolus Rex Iudaeorum ${ }^{103}$.

Per effetto di più eventi tra loro convergenti, l'epilogo della vicenda si consumò tra il 1745 ed il 1747. Sotto il profilo politico, il Segretario di Stato Montealegre,

\footnotetext{
${ }^{102}$ Ajello, 1976, p. 653.

${ }^{103}$ L'aneddoto è documentato da Lacerenza, 2017, p. 145. Carlo sarebbe stato soprannominato da alcuni Infans Carolus Rex ludaeorum, a somiglianza di lesus Nazarenus Rex Iudaeorum, e da altri Infans Corolus Iudaeorum, con un gioco di parole che faceva riferimento al titolo ufficiale di Infans Hispaniarum.
} 
che già a partire dall'agosto del 1745 era stato di fatto esautorato del proprio potere, fu sostituito da Giovanni Fogliani nel giugno 1746. In perfetta concomitanza con tale avvicendamento, il 18 settembre 1746 Carlo adottò la risoluzione di revocare i privilegi concessi alla Nazione ebrea: decisione che preannunciò il definitivo bando di espulsione emanato il 30 luglio 1747.

Sul piano delle affermazioni esplicite, il governo motivava la revoca sostenendo che il proclama del 1740 aveva «deluso le [...] speranze» dei riformatori, perché gli ebrei erano

qua venuti senza fondi, senza capitale, senza credito, e non con altra qualità, o talenti per il negozio, che quelli della consueta loro avidità di arricchirsi per via delle straordinarie usure, e di altri illegittimi mezzi, così che sono anzi riusciti di forte ostacolo all'accrescimento del commercio, e d'incommodo, di peso, e di scandalo a' nostri Popoli. L'esperimento che di tutto questo abbiamo fatto nel corso di sei anni, ci scioglie dall'obbligo di ogni graziosa promessa i nostri obblighi ${ }^{104}$.

Ben al di là della motivazione espressa, era chiaro che la revoca del proclama fosse lo specchio del mutamento politico-istituzionale in atto nel Regno: una vera e propria parabola era stata percorsa dal governo borbonico, dalle audaci iniziative di ammodernamento varate nei primi anni che avevano rappresentato la fase ascendente sino agli epiloghi delle più importanti riforme che, tra il 1746 ed il 1747, rappresentarono il culmine della fase discendente.

In questa prospettiva, l'esame delle controverse vicende intorno agli ebrei rende evidente che quegli eventi seguirono un andamento che, nei tempi e nei modi, risulta sovrapponibile alle dinamiche che interessarono il Supremo Magistrato del Commercio. II parallelismo esistente tra le due vicende storiche fa emergere in filigrana la complessa orditura dei mutevoli assetti di potere che caratterizzarono il governo carolino.

Entrambe le iniziative, infatti, erano nate nell'humus fertile creatosi a Napoli all'indomani dell'arrivo di Carlo di Borbone e che, riuscì a dare i suoi frutti più importanti proprio tra il 1739 ed il 1740. Le idee dei novatores avevano trovato, nel nuovo contesto politico, concrete possibilità di realizzazione grazie anche al mutamento del rapporto delle forze internazionali che aveva avuto ripercussioni rilevanti specialmente nei confronti dalle grandi potenze marittime.

Il complesso di quelle condizioni aveva consentito di sacrificare gli interessi, pur sempre fortissimi, dei ceti tradizionalmente privilegiati e di tentare un'operazione a dir poco complessa in una società bloccata qual era quella napoletana: intaccare il nocciolo duro, in larga parte incancrenito, delle consolidate posizioni di potere. Le prospettive di competitività a livello internazionale avevano imposto al Regno di compiere un vero e proprio salto, ma presto, evidentemente, questo si era rivelato troppo lungo.

L'evidente congiuntura temporale che segna l'editto istitutivo del Supremo Ma-

${ }^{104}$ Prammatica VII, in Giustiniani, 1804, pp. 110-111. 
gistrato del Commercio ed il proclama di riammissione degli ebrei testimonia che entrambe le iniziative furono il frutto del prevalere del medesimo indirizzo progressista assunto nei primi anni del governo carolino.

Il collegamento esistente, sin dal nascere, tra le due iniziative è testualmente dimostrato dalla corrispondenza intercorso tra Montealegre e Contegna tra l'8 ed il 12 ottobre 1739, allorquando il marchese aveva incaricato il sacerdote di Arienzo dell'esame congiunto di entrambe le questioni ${ }^{105}$.

Pietro Contegna e Celestino Galiani, supportati dal Segretario di Stato marchese e poi duca di Salas, diventavano protagonisti di una stagione di riforme che dimostra quanto vantaggiosi possano essere gli effetti della sinergia tra cultura e potere politico.

Piena era la fiducia riposta dai riformatori in entrambe le iniziative che erano legate significativamente in un'unica trama. II capo VI dell'editto di riammissione degli ebrei reca conferma di quest'impostazione: nel concedere alla Nazione ebrea «un Ministro Delegato nella fedelissima Città di Napoli, e due nel Regno di Sicilia» specificava che lo stesso dovesse essere "uno delli Ministri del Supremo Magistrato del Commercio» ${ }^{106}$. Sarebbe spettato soltanto a costoro la giurisdizione su tutte le

Cause, che sorgeranno frà un Cristiano, ed un Ebreo, e tutte quelle Cause, che si agiteranno fra Ebreo, ed Ebreo purché si tratti di qualche delitto, che meriti pena maggiore della relegazione, o dell'esilio ${ }^{107}$.

La felice orditura tessuta dai riformatori tra il 1739 ed il 1740 evidenzia l'esistenza di un fronte compatto di intellettuali che, mosso dall'audace progetto di ammodernare lo Stato nel suo complesso, trova nel governo un decisivo interprete di quell'indirizzo politico. Non a caso, quello straordinario parallelismo $d^{\prime}$ intenti e di iniziative le iniziative riformistiche lungo tutto il loro iter fino ad un comune infelice epilogo che fu la dimostrazione indiretta della loro piena validità. Gli effetti di entrambe le innovazioni furono annullati, tra il 1746 ed il 1747, a causa del prevalere di quelle forze particolaristiche e cetuali che proprio quelle riforme avevano indicato come nemici di qualsiasi assetto statale moderno ed aggiornato.

Nel 1746, infatti, il Supremo Magistrato del Commercio di Napoli fu oggetto di una vera e propria "controriforma» che, a distanza di soli sette anni dalla sua istituzione, strozzò la magistratura commerciale, limitandone la giurisdizione alle sole cause riguardanti il commercio "esterno». La decisione, maturata il primo agosto 1746 in un clima politicamente ed economicamente stressato dalla guerra di successione austriaca, era stata motivata sul presupposto del presunto in-

\footnotetext{
${ }^{105}$ ASN, Ministero degli affari esteri, 4400, 9/4.

${ }^{106}$ Cfr. Prammatica VI, De expulsione Hebraeorum sive ludeorum, in Giustiniani, 1804, p. 105.

107 Ibidem.
} 
successo della magistratura commerciale e della volontà di destinare una "grazia speciale» ai "fedelissimi sudditi» ${ }^{108}$, ma, di fatto, essa era stata una risposta coerente alle richieste della nobiltà e della borghesia di toga, la cui ostilità nei confronti del progetto di ammodernamento giudiziario carolino, era stata ben manifesta, sin dal suo avvio, tanto al Sovrano quanto al Segretario di Stato José Joaquín de Montealegre.

Nel nuovo contesto politico minacciato dalle pretese austriache, quell'onda montante d'insofferenza aveva assunto contorni preoccupanti che avevano imposto una decisa precisa di posizione, definitivamente compiutasi con la «controriforma» del 1746. Essa fu la chiara manifestazione che nulla dovesse essere cambiato e che fosse indispensabile tornare al passato, piuttosto che guardare al futuro. Ridotti alla stregua dei tanti fori particolari già esistenti nel Regno, il Supremo Magistrato e i pochi Consolati sopravvissuti divennero l'ennesima manifestazione di particolarismo giuridico e giudiziario: un processo di complessivo snaturamento della riforma poteva dirsi compiuto.

Allo stesso modo, le dinamiche che interessarono la comunità ebraica trovarono il loro sostanziale e definitivo epilogo nel 1747, allorquando l'ordine di espulsione degli ebrei dal Regno segnò un decisivo e significativo ritorno al passato.

Sul piano delle idealità che sempre sostengono i fatti, entrambe le iniziative avevano dimostrato che, per promuovere la produttività ed il commercio, bisognava incidere contemporaneamente sul tessuto sociale, giuridico, politico ed istituzionale, ponendo in essere un'audace manovra di virata. Sul piano dei fatti che non sempre corrispondono alle idealità, esse rivelarono che, senza una base di consenso e sostegno politico, qualsiasi progetto, per quanto fondato e ambizioso possa essere, è destinato a produrre frutti effimeri.

\section{Fondi di Archivio}

*Abbreviazioni:

ASN = Archivio di Stato di Napoli

$\mathrm{BCR}=$ Biblioteca Corsiniana di Roma

SNSP = Biblioteca della Società Napoletana di Storia Patria

ASN, Casa Reale Antica, f. 912, Piano delle facoltà, giurisdizioni e regole di governo colle quali dovranno regolarsi il Supremo Magistrato del Commercio di questo Regno, li Consolati, Giudici e Corti al predetto magistrato sottoposte

ASN, Ministero degli affari esteri, 4400, 1/3, Circa gli Ebrei

ASN, Ministero degli affari esteri, 4400, 9/2, Lettera di Montealegre a Contegna dell'8 ottobre 1739

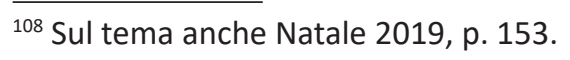


ASN, Ministero degli esteri, 4400, 9/4, Lettera autografa di Pietro Contegna del 12 ottobre 1739, in risposta alla lettera di Montealegre dell'8 ottobre 1739

ASN, Ministero degli affari esteri, 4401,6/5, Riflessioni sulle due scritture della Romana Inquisizione intorno all'introduzione degli ebrei nelli Regni di Napoli e di Sicilia

ASN, Ministero degli affari esteri, 4401, 6/9, Riflessioni su la lettera venuta di Roma in proposito degli ebrei

ASN, Ministero degli affari esteri, 4401, 6/3, Memoria del Cardinal Firrao inviata da Roma il 22 gennaio 1740

ASN, Ministero degli affari esteri, 4401, 6/4, Risposta del Contegna del 30 gennaio 1740 al cardinal Firrao

ASN, Ministero degli affari esteri, 4402, Foglio del Signor Cardinale Arcivescovo contenente alcune osservazioni sopra il proclama o sia banno, col quale fu permesso agli ebrei di venire a traficare nel regno delle Due Sicilie con istabilirvi il loro domicilio

ASN, Ministero degli affari esteri, 4402, Galiani a Montealegre, 1 giugno 1742

ASN, Ministero degli affari esteri, 4402, Tanucci al Re, 11 giugno 1742

BCR, cod. cors. 1891 ms. 44.E.1, Lettera autografa del 29 luglio 1724 scritta da A. Niccolini a Bottari

SNSP, ms. XXXI B-1, Parere teologico sopra alcuni punti appartenenti all'introduzione degli Ebrei nei due regni di Napoli e Sicilia

SNSP, ms. XXI d30, Pareri della Giunta di Commercio

\section{Bibliografia}

Abulafia D. S. H., 1998: The role of the Jews in the cultural life of the Aragonese kingdom of Naples, in Bucaria N. (ed.), Gli ebrei in Sicilia dal tardoantico al Medioevo, Studi in onore di Mons. B. Rocco, Palermo, Flaccovio

Abulafia, D.S.H.,1997:Ilmezzogiornopeninsularedaibizantiniall'espulsione(1541), in Gli Ebrei in Italia. Dall'alto Medioevo all'età dei ghetti, I, Torino, Einaudi

Ajello R., 1976: La vita politica napoletana sotto Carlo di Borbone. La "fondazione ed il tempo eroico "della dinastia ", in Storia di Napoli, VII, Napoli, Edizioni Scientifiche Italiane

Ajello R., 1980: Dal Giurisdizionalismo all'lluminismo nelle Sicilie: Pietro Contegna, in Studi in memoria di Ernesto Pontieri, Archivio Storico per le Province Napoletane, Terza Serie, Vol. XIX, Napoli

Ajello R., 1998: Una società anomala. II programma e la sconfitta della nobiltà napoletana in due memoriali cinquecenteschi, Napoli, Edizioni Scientifiche Italiane 
Andreoni L., 2018: A chi appartengono le case del ghetto di Ancona? Ebrei e catasto tra cultura illuministica e polemica antiebraica, in "Proposte e ricerche, Economia e società nella storia dell'Italia centrale", 41

Andreoni L., Gasperoni M., 2018: Ebrei e proprietà nelle città dell'Italia centrale: un'introduzione, in "Proposte e ricerche, Economia e società nella storia dell'Italia centrale", 41

Andreoni L., 2019: «Una nazione in commercio». Ebrei di Ancona, traffici adriatici e pratiche mercantili in età moderna, Milano, Angeli

Artom E., 1950, Gli Ebrei nel Settecento, in "Rassegna mensile di Israel", XVI, 1

Ascione I., 2000: Al servizio dell'Infante Duca. Bernardo Tanucci alla corte di Carlo di Borbone nell'estate del 1733, in "Frontiera d'Europa", 1\2000

Bartolucci G., 2007: La Repubblica ebraica di Carlo Sigonio. Modelli politici dell'età moderna, Firenze, L. S. Olschki

Boari M., 1992: Usura - Diritto intermedio, in Enciclopedia del diritto, XLVI, Milano

Caffiero M., 2014: Storia degli ebrei nell'Italia moderna. Dal Rinascimento alla restaurazione, Roma, Carocci

Caridi G., 2011: Dall'investitura al concordato: contrasti giurisdizionali tra Napoli e Santa Sede nei primi anni del regno di Carlo di Borbone, in "Mediterranea", 23

Cassandro M., 1984: Gli ebrei di Livorno nel Seicento. Aspetti economici e sociali, in "La Rassegna Mensile di Israel", Vol. 50, n. 9\12

Colafemmina C., 2013: Gli ebrei in Puglia sotto Ferdinando il Cattolico (1503 1516), in Lacerenza G. (ed.), 1510 - 2010 Cinquecentenario dell'espulsione degli ebrei dall'Italia meridionale. Atti del Convegno internazionale, Napoli, Università degli Studi di Napoli "L'Orientale"

Ciancio C., 2012: Mercanti in toga. I Tribunali di Commercio nel Regno d'Italia (1861-1888), Bologna, Patron

Conde y Delgado de Molina R., 1991: La expulsíon de los judíos de la Corona de Aragón. Documentos para su estudio, Saragozza

Cernigliaro A., 1984: Sovranità e feudo nel Regno di Napoli (1505-1557), Napoli, Jovene

Cernigliaro A., 2006: La "polizia del Regno". Per moderare la manomorta ecclesiastica, in "Archivio Storico per le Province Napoletane"

Cernigliaro A., 1988: Patriae leges privatae rationes. Profili giuridico-istituzionali del Cinquecento napoletano, Napoli, Jovene

Dani, A., 2021: Cittadinanze e appartenenze comunitarie. Appunti sui territori toscani e pontifici di Antico regime, Roma, Collana di Studi di Storia del diritto medievale e moderno, Historia et ius

Del Bagno I., 2000: I/ duello "certamen licitum»? Problemi giuridici e reviviscenza settecentesca nel regno di Napoli, in "Frontiera D'Europa", 2 
Del Treppo M., 1995: Realtà, mito e memoria di Napoli aragonese, in Zilli I. (ed), Fra spazio e tempo. Studi in onore di L. De Rosa, I, Napoli, Edizioni Scientifiche Italiane

D'Ippolito F. E., 2006: Disincagliare l'economia per "governare la nazione", in "Studi Veneziani", LII

Dubin L. C., 1999: The Port Jews of Habsburg Trieste. Absolutist Politics and Enlightenment Culture, Stanford, University Press

Dubin L. C., 2010: Ebrei di porto nella Trieste asburgica. Politica assolutista e cultura dell'Illuminismo, Gorizia, LEG

Edigati D., 2020: La tolleranza per privilegio nell'Italia di Antico Regime. II caso degli ebrei e dei cristiani orientali, in "Archivio Giuridico Filippo Serafini", 152, 3

Edigati D., 2021: La "Livornina» e i confini della tolleranza religiosa nella Toscana d'età moderna, in Edigati D., Tira A. (ed.), Le minoranze religiose nel diritto italiano ed europeo. Esperienze del passato e problematiche contemporanee, Torino, Giappichelli

Eisentadt S. N., 1993: Civiltà ebraica. L'esperienza storica degli Ebrei in una prospettiva comparativa, Roma, Donzelli

Ferorelli N., 1915: Gli Ebrei nell'Italia meridionale dall'età romana al secolo XVIII, Torino, a cura della rivista II vessillo israelitico

Ferrone V., 1982: Scienza Natura Religione. Mondo newtoniano e cultura italiana del primo Settecento, Napoli, Jovene

Fiume G., 2009: Schiavitù mediterranee. Corsari, rinnegati e santi di età moderna, Milano, Mondadori

Foa A., 2001: Ebrei in Europa. Dalla Peste Nera all'emancipazione, XIV-XIX secolo, Roma-Bari, Laterza

Frattarelli Fischer L., 2008: Vivere fuori dal ghetto. Ebrei a Pisa e Livorno (secoli XVI-XVIII), Torino, Zamorani

Frattarelli Fischer L., 2019: Le Leggi Livornine. 1591-1593, Livorno, Media Print

Galasso C., 2002: Alle origini di una comunità. Ebree ed ebrei a Livorno nel Seicento, Firenze, L. S. Olschki

Galiani C., Grandi G., 1989: Carteggio (1714-1729), a cura di F. Palladino A L. Simonutti, Firenze, L. S. Olschki

Gamba C., 2003: Licita usura. Giuristi e moralisti tra Medioevo ed Età moderna, Roma, Viella

Gatti C., 2008: Tra demografia e storia sociale. Gli Ebrei di Trieste nel Settecento, Trieste, EUT

Genovesi A., 2005: Delle lezioni di commercio o sia di economia civile con elementi di commercio, a cura di M. L. Perna, Napoli, nella sede dell'Istituto

Giura V., 1978: Gli ebrei e la ripresa economica del Regno di Napoli: 1740-1747, 
Napoli, Institut International d'Histoire de la Banque

Giura V., 1984: Gli ebrei nel Regno di Napoli tra Aragona e Spagna, in Giura V. (ed), Storie di minoranze. Ebrei, greci, albanesi nel Regno di Napoli, Napoli, Edizioni Scientifiche Italiane

Giustiniani L., 1804: Nuova collezione delle prammatiche del Regno di Napoli, IV, Napoli, Stamperia Simoniana

Graziani Secchieri L., 2018: Da chazakah a jus kazakà a proprietà: l'origine del diritto d'inquilinato perpetuo in ghetto nelle radici normative ebraiche. II caso di Ferrara, in "Proposte e ricerche. Economia e società nella storia dell'Italia centrale", 41

Hertzberg A., 1968: The French enlightenment and the Jews, New York - London, Columbia University Press

Katz J., 1973: Out of the Ghetto. The Social Background of Jewish Emancipation, 1770-1870, Cambridge (Mass.), Harvard University Press

Lacerenza G., 2002: Lo spazio dell'ebreo. Insediamenti e cultura ebraica a Napoli (secoli XV - XVI), in L. Barletta (ed), Integrazione ed emarginazione, Circuiti e modelli: Italia e Spagna nei secoli XV-XVIII, Napoli, CUEN

Lacerenza G. (ed), 2013: 1510-2010 Cinquecentenario dell'espulsione degli ebrei dall'Italia meridionale. Atti del Convegno internazionale, Napoli, Università degli Studi di Napoli "L’Orientale"

Lacerenza G., 2017: Carolus Rex ludaeorum? Per una rilettura dei rapporti fra Carlo IIl e gli ebrei, in L. Cerullo (ed), Carlo di Borbone: un sovrano nel mosaico culturale dell'Europa, Napoli, Università degli Studi di Napoli “L'Orientale"

Le Goff J., 1987 : La bourse et la vie. Economie et religion au Moyen Age, Paris 1986, trad. it. Roma-Bari, Laterza

Luongo D., 2008a: Consensus gentium. Criteri di legittimazione dell'ordine giuridico moderno, I, Oltre il consenso metafisico, Napoli, Arte tipografica

Luongo D., 2008b: Consensus gentium. Criteri di legittimazione dell'ordine giuridico moderno, II, Verso il fondamento sociale del diritto, Napoli, Arte tipografica

Luongo D., 2018: /l giurisdizionalismo dei moderni. Polemiche anticuraliastiche nella Napoli del Preilluminismo, Torino, Giappichelli

Luzzatto Voghera G., 2000: Percorsi della cultura ebraica in età moderna, in P. Reinach Sabbadini (ed), La cultura ebraica, Torino, Einaudi

Macario F., Manna A. (ed), 2002: Mercato del credito e usura, Milano, Giuffrè

Mastroianni F., 1972: Un amico di G. B. Vico nella storia dei cappuccini di Napoli: B. M. Giacco (1672-1744), Napoli, Editrice Campania Serafica

Militi M., 2013: Gli ebrei e l'Età dei Lumi tra "emancipazione"e "rigenerazione". Una panoramica storiografica, in "Eurostudium" 
Moulinas R., 1981 : Les juifs du pape en France, les communautés d'Avignon et du comtat venaissin aux 17 e et 18 e siècles, Paris, Privat

Natale M., 2002: Eclettismo teoretico e pragmatismo alle origini delle riforme illuministiche: I'autobiografia di Celestino Galiani, in "Frontiera d'Europa", 1 e 2

Natale M., 2014: Sui piatti della bilancia. Le magistrature del commercio a Napoli (1690 -1746), Milano, Giuffrè

Natale M., 2019: Nuova forma e nuove fonti per il Supremo Magistrato del commercio, in "Rivista di Storia del diritto italiano", XCII, 1

Nelson B., 1967: Usura e cristianesimo. Per una storia della genesi dell'etica moderna, Firenze, Sansoni

Nicolini F., 1951: Un grande educatore italiano: Celestino Galiani, Napoli, F. Giannini e Figli

Novi Chavarria E., 2020: Il confessore alla Corte di Carlo, in A. M. Rao (ed), Corte e cerimoniale di Carlo di Borbone a Napoli, Napoli, FedOAPress

Origlia G. P., 1753: Istoria dello studio di Napoli, Napoli

Pérez J., 2005: Los Judíos en España, Madrid, Marcial Pons Historia

Pilati R., 2001: Del commercio: Gregorio Grimaldi ed il riformismo napoletano nella prima età borbonica, in "Frontiera d'Europa"

Ravid B. C. I., 1978: Economics and toleration in seventeenth century Venice. The background and context of the Discorso of Simone Luzzatto, Jerusalem and New York

Ravid B. C. I., 2018 (1a 2003): Studies on the Jews of Venice, 1382 - 1797, Oxford, Routledge

Rosenberg H., 1935: Alcuni documenti riguardanti i marrani portoghesi in Ancona, in "La Rassegna Mensile di Israel"

Santarelli U., 1992: Mercanti e società tra mercanti, Torino, Giappichelli

Santarelli U., 2005: Sei lezioni sull'usura, Pisa, Servizio editoriale universitario

Simonsohn S., 2017: Gli ebrei a Roma e nello Stato Pontificio da Paolo IV a Pio IX. Un quadro d'insieme, in "Materia Judaica", XXII

Stock M., 1977: L'editto di espulsione degli ebrei dal Regno di Napoli (1510) e la loro breve riammissione nel Settecento, in "La Rassegna Mensile di Israel", terza serie, 43, n. $1 \backslash 2$

Storti Storchi C., 2012: Alcune considerazioni sul trattamento dello straniero in età medievale e moderna tra flessibilità e pragmatismo, in M. Meccarelli, P. Palchetti, C. Sotis (ed), lus peregrinandi. II fenomeno migratorio tra diritti fondamentali, esercizio della sovranità e dinamiche di esclusione, Macerata, EUM

Tasmani G, 1999: /l giudaismo nell'età moderna e contemporanea, in G. Filoramo (ed), Ebraismo, Roma-Bari, Laterza 
Tita M., 2008: Processi per usura. Ideologie giuridiche e soluzioni giudiziarie tra Sette e Ottocento, Napoli, Edizioni Scientifiche Italiane

Tita M., 2012: /l giudizio dei Pari. La giurisdizione commerciale a Roma e a Napoli tra Sette e Ottocento, Campobasso, AGR

Toaff A. S., 1965: La giurisdizione autonoma degli ebrei di Livorno e la controversia con R. Ja'acob Sasportas (1680), in "La Rassegna Mensile di Israel”, Vol. 31, No. 6 ,

Todeschini G., 2003: Proprietà ebraica, potere cristiano, storia economica: la "sicurezza nella possessione dei propri beni" come forma della socialità, in "Parolechiave", XXX

Trifone G. P., 2013: Contegna Pietro, in I. Birocchi, E. Cortese, A. Mattone, M. N. Miletti (ed), Dizionario biografico dei giuristi italiani (XII-XX secolo), I, Bologna, II Mulino 
